\title{
Checking Safety Properties using Compositional Reachability Analysis ${ }^{1}$
}

\author{
Shing Chi Cheung \\ Department of Computer Science \\ Hong Kong University of Science and \\ Technology, Clear Water Bay, Hong Kong
}

\author{
Jeff Kramer \\ Department of Computing \\ Imperial College of Science, Technology and \\ Medicine, London SW7 2BZ, UK
}

\begin{abstract}
The software architecture of a distributed program can be represented by a hierarchical composition of subsystems, with interacting processes at the leaves of the hierarchy. Compositional reachability analysis (CRA) is a promising state reduction technique which can be automated and used in stages to derive the overall behaviour of a distributed program based on its architecture. CRA is particularly suitable for the analysis of programs that are subject to evolutionary change. When a program evolves, only the behaviours of those subsystems affected by the change need be reevaluated. The technique however has a limitation. The properties available for analysis are constrained by the set of actions that remain globally observable. Properties involving actions encapsulated by subsystems may therefore not be analysed. In this paper, we enhance the CRA technique to check safety properties which may contain actions that are not globally observable. To achieve this, the state machine model is augmented with a special trap state labelled as $\pi$. We propose a scheme to transform, in stages, a property that involves hidden actions to one that involves only globally observable actions. The enhanced technique also includes a mechanism aiming at reducing the debugging effort. The technique is illustrated using a gas station system example.
\end{abstract}

\footnotetext{
1 This paper is an invited submission to TOSEM, a revised and extended version of [Cheung96a].
} 


\section{Introduction}

\subsection{Background}

Distributed processing is used to provide computing support for many diverse applications. Many of these applications are complex and critical; an error can have catastrophic consequences. Behaviour analysis is one of the techniques that can help to discover defects and check if a program performs as intended. However, concurrent and distributed programs are generally more complex to analyse than their sequential counterparts. Even for small programs, analysis of their behaviour is impractical without the support of an effective automated technique.

Static analysis techniques for concurrent and distributed programs can be used to verify two classes of property: safety and liveness [Alpern85, Corbett95, Manna95]. A safety property asserts that the program never enters an undesirable state. For example, mutual exclusion is a safety property that specifies the absence of a program state where a common resource is simultaneously accessed by more than one client. A liveness property asserts that a program eventually enters a desirable state. For example, freedom from starvation is a liveness property; it says that a program state, where some request is served, will finally be entered.

In this paper, we focus our discussion on the provision of an effective technique for checking safety properties that characterise undesirable execution sequences of a program. These safety properties can be specified in the form of regular expressions or deterministic finite-state machines [Dwyer94, Olender90]. The two formalisms are interchangeable. For the ease of understanding, we use the formalism of state machines. State machines that specify safety properties are called property automata. Each property automaton specifies the set of feasible execution sequences over the actions (transitions) that correspond to a safety property of interest. For example, the property automaton in Figure 1 asserts that (i) no execution of action update can occur unless preceded by an execution of action lock and (ii) every lock action is succeeded by an update and an unlock action.

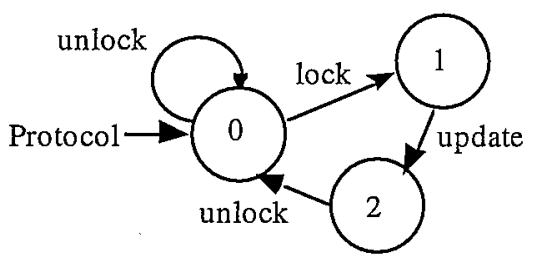

Figure 1: A Property Automaton 
The software architecture of a distributed program can be represented by an hierarchical composition of subsystems, with interacting (primitive) processes at the leaves of the hierarchy. Behaviour of a primitive process can be modelled similarly, as a state machine whose transitions are labelled by the activities it can perform. In this paper, we assume that the behaviour of each primitive process can be expressed in a finite state deterministic machine. Composite processes appear at the intermediate nodes of the hierarchy. Each composite process is a subsystem formed by a collection of processes; these processes can be either primitive or composite.

Static analysis is an approach to program behaviour verification without execution. The approach is particularly useful in identifying program design errors prior to implementation. Various static analysis techniques have been proposed for checking properties of distributed programs. These include techniques such as symbolic model checking [McMillan93], inequality necessary conditions analysis [Avrunin91, Corbett95], data flow analysis [Cheung94, Dwyer94, Masticola91] and explicit state enumeration [Cheung96b, Godefroid91, Holzmann91, Long89, Taylor83, Valmari93]. A number of recent studies have applied static analysis techniques to verify various types of concurrent application [Anderson96, Corbett96, Dwyer97, Holzmann97, Juval98, Mascarenhas98, Naumovich97, Young95].

Symbolic model checking has been widely used to verify designs of digital circuits against properties expressed in branching time temporal logic CTL. The state space is represented symbolically by a logical formula captured using a Binary Decision Diagram (BDD). The technique works well for hardware designs with regular logical structures. For such systems, BDD representations can reduce the state space from exponential order of the number of state variables to linear. However, it is less likely to achieve similar reductions in software specifications whose logical structures are less regular. Techniques using explicit state enumeration were found to be more effective than that using BDD representation in the verification of a safety injection component of a naval system [Bharadwaj97]. This is because a BDD represents all possible states while explicit state enumeration techniques consider only reachable states. Attempts are being made to adapt symbolic model checking for software systems [Anderson96]. In the inequality necessary conditions analysis[Avrunin91, Corbett95], the necessary conditions for the existence of an execution trace that violates a specified property are expressed using a set of inequalities. These inequalities are then solved using standard integer linear programming packages. Non-existence of solutions guarantees the satisfiability of the property while existence of solutions yield an inconclusive result. The 
technique has the advantage that the number of inequalities is essentially linear to the number of concurrent processes in a program. However, integer linear programming problems are generally NP-hard and the standard techniques involved are potentially exponential. Data flow analysis techniques adopt a different approach. By approximating the execution model of a program, properties can be efficiently checked using a polynomial algorithm. However the conclusion thus obtained is usually either complete or sound but not both [Cheung94, Dwyer94, Masticola91, Reif90].

Analysis using explicit state enumeration is conducted by constructing an equivalent state machine of the program against which properties can be checked. It is generally known as reachability analysis. A major problem of reachability analysis is that the search space involved can expand exponentially with the increase in the number of concurrent processes. Reduction techniques have been proposed to alleviate the problem by not having to construct the entire state graph. Reduction techniques can be broadly classified into two categories: reduction by partial ordering and reduction by compositional minimisation. In the former category, reduction is achieved by avoiding the generation of all paths formed by the interleaving of the same set of transitions [Godefroid91, Holzmann92, Valmari91]. In the latter category, reduction is achieved by intermediate minimisation of subsystems [Malhotra88, Sabnani89, Tai93a, Tai93b, Yeh91]. Reduction techniques in this category are known as compositional reachability analysis (CRA). We adopt this latter approach as it is effective, amenable to automation and closely matches the software architecture (structure) of the program design.

\subsection{Related Work of Compositional Reachability Analysis}

Compositional reachability analysis (CRA) techniques were originally proposed to remedy the problem of traditional reachability analysis techniques [Peterson81, Pezzè95, Taylor83] which compose the global system representation in a single step. Yeh [Yeh93a] described several case studies which suggested similar performance between a technique of compositional reachability analysis and that of constrained expressions [Avrunin91]. Sabnani [Sabnani89] described an experiment applying compositional reachability analysis to the Q.931 protocol. They found that the intermediate state space graphs generated never exceeded 1,000 states although the global state space graph given by traditional reachability analysis of the protocol contained over 60,000 states. A similar observation has also been made by [Giannakopoulou98] in the case study of a reliable multicast transport protocol that contains over 96,000 states and 672,000 transitions. The intermediate state space graphs generated by the CRA never exceeded 400 states. Furthermore, CRA techniques are particularly suitable to 
analyse programs that are likely to evolve. The techniques help localise the effect of change. When changes are applied to a program, only the state machines of those subsystems that are affected by the changes need be re-computed.

Although CRA techniques have advantages over traditional techniques of reachability analysis, the system representation generated cannot be utilised to validate behavioural properties involving actions that are not globally observable. Verification has to be restricted to only those properties formed by globally observable actions. However, a complex program typically involves many sophisticated interactions between multiple subsystems. These subsystems can be independently developed or extracted from software libraries. Each assumes a set of predefined communicating protocols at its interface. For a subsystem to function properly, its neighbours must respect its protocols. These protocols can therefore be conceived as the subsystem properties that have to be satisfied in the composite system. Checking the satisfaction of all these properties in the global context may therefore lead to the need to expose many or all subsystem interactions even though many of them may be irrelevant to the abstract view of the system. This contradicts the key philosophy of abstraction and jeopardises the effectiveness of CRA techniques when applied to complex programs.

In this paper, we enhance the CRA technique with a mechanism to validate safety properties without the need for making the involved actions globally observable. These properties are violated when some subsystems, within the context of a distributed application, can perform execution sequences not acceptable to the specified property automata. If no violation of safety properties is detected, the analysis constructs a global labelled transition system observationally equivalent [Milner89a] to that constructed using conventional CRA techniques; otherwise it indicates which safety properties are violated and how the violations can occur. We have found no similar work providing this feature in the framework of CRA.

The proposed mechanism is complementary to state space reduction techniques for CRA [Cheung96b, Graf90]. Cheung proposed a method to alleviate state explosion encountered in CRA by using context constraints automatically derived from the environment of each subsystem. The output delivered by the method is a state machine representing the global behaviour of the program being analysed. However, developers generally wish to validate desirable program properties in addition to the generation of a global state machine. To check safety properties using the mechanisms of CRA, we add a special trap state to the state machine model. The trap state, labelled as $\pi$, identifies undesirable execution sequences of a program. The use of trap states for checking behavioural properties has been proposed in 
some data flow analysis techniques, such as CECIL [Olender90] and FLAVERS [Dwyer94], but these techniques do not make use of the given design hierarchy. Trap states can also be utilised to detect erroneous program behaviour constraints specified by users [Cheung95].

\subsection{Paper Outline}

In the next section, we introduce labelled transition systems and present a gas station system, which is used as an example in our discussions. Section 3 presents a technique to detect and locate violation of safety properties in the framework of CRA, illustrating experience of its use on the example. Section 4 gives an account of a mechanism which reduces the effort of debugging in the compositional framework. This is followed by performance evaluation and conclusions in sections 5 and 6 , respectively.

\section{Background}

\subsection{Labelled Transition Systems}

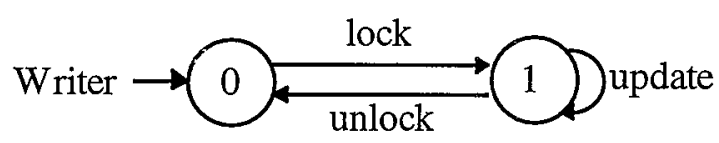

\section{Figure 2: An LTS Description of a Writer}

A labelled transition system (LTS) can be used to model the behaviour of processes in a distributed program. An LTS contains all the states that the process may reach and all the transitions that it may perform. For instance, Figure 2 represents an LTS describing a writer that repeatedly updates information in a shared resource. The Writer must first go from state 0 to 1 to lock the shared resource. Unlocking the resource results in the transition back to state 0 .

Actions performed by a program are given the same label if there is no need to distinguish them from one another. The set of actions relevant for a particular behavioural description of a process $P$ is called its alphabet [Hoare85], denoted as $\alpha P$. In the above example, the alphabet of Writer equals \{lock, unlock, update\}. The alphabet is a permanent predefined property of a process. Logically, a process may only perform actions belonging to its alphabet. For example, Writer cannot perform an action read which is outside its alphabet. However, a process might never perform an action in its alphabet. An alphabet is so chosen as to make analysis tractable. This involves decisions to ignore many other properties and actions considered to be of lesser interest. A process may perform some activities which 
cannot be influenced by its environment. These activities are labelled by an internal action which is represented by the symbol $\tau$. The presented LTS computational model has been widely used in the literature for specifying and analysing distributed programs [Clarke89, Ghezzi91, Kemppainen92, Rabinovich92, Valmari92]. Formally, an LTS of a process $P$ is a quadruple $\langle S, A, \Delta, q\rangle$, where

(i) $S$ is a set of states;

(ii) $A=\alpha P \cup\{\tau\}$, where $\alpha P$ is communicating alphabet of $P$ which does not contain the internal action $\tau$;

(iii) $\Delta \subseteq S \times A \times S$ denotes a transition relation;

(iv) $q$ is a state in $S$ which indicates the initial state of $P$.

An LTS of $P=\langle S, A, \Delta, q\rangle$ transits into another LTS of $P^{\prime}=\left\langle S, A, \Delta, q^{\prime}\right\rangle$ with an action $a$ $\in A$ if and only if $\left(q, a, q^{\prime}\right) \in \Delta$ and $q^{\prime} \neq \pi$, where $\pi$ is a trap state to be discussed further below. That is,

$$
<S, A, \Delta, q>\stackrel{a}{\longrightarrow}<S, A, \Delta, q^{\prime}>\operatorname{iff}\left(q, a, q^{\prime}\right) \in \Delta \text { and } q^{\prime} \neq \pi
$$

Since there is a one-to-one mapping between a process $P$ and its LTS, the term process and LTS are used interchangeably. The above statement may also be rewritten as:

$$
P \stackrel{a}{\longrightarrow} P^{\prime} \text { iff }\left(q, a, q^{\prime}\right) \in \Delta \text { and } q^{\prime} \neq \pi
$$

The trap state $\pi$ is introduced as an error state at which specified safety properties are violated. A process that is trapped at the $\pi$ state can participate into no further actions. Its behaviour is represented by an LTS $\langle\{\pi\}, \Sigma, \varnothing, \pi\rangle$, where $\Sigma$ represents the universal set of actions. For convenience, we use $\Pi$ to denote a trapped process. Thus a process $P=<S, A, \Delta$, $q>$ transits into $\Pi$ if it executes a transition $(q, a, \pi)$ in $\Delta$. For instance, Figure 3 represents a Writer that transits into $\Pi$ after executing update at state 0 . Hence, Writer $\stackrel{\text { update }}{\longrightarrow} \Pi$.

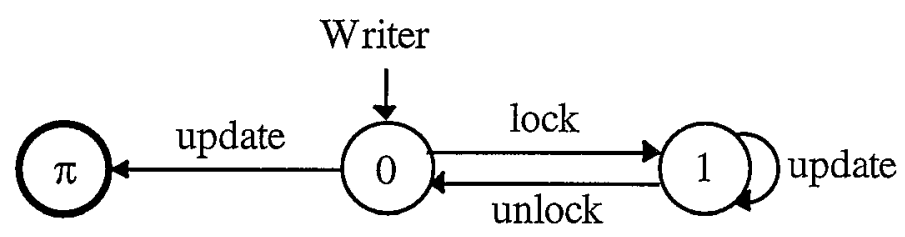

Figure 3: A Writer with a Trap State

Behaviour of a process can be observed by means of its execution traces. An execution trace 
of a process is a sequence of actions that it can perform. For example, the sequence <lock, update, unlock $>$ is a trace of Writer in Figure 2 and 3. The set of possible traces of a process $P$ is denoted by $\operatorname{tr}(P)$, whose formal definition can be found in the work of Hoare [Hoare85]. A trace is said to be trapping if its execution leads a process to $\Pi$; otherwise it is nontrapping. A process is said to be trappable if it contains some trapping traces; otherwise it is nontrappable.

Observability of actions in a process can be controlled by a restriction operator " $\uparrow " P \uparrow L$ represents the process projected from $P$ in which only the actions in set $L$ are observable. The restriction operator ensures that $P$ has trapping traces if and only if $P \uparrow L$ has. Rules (1) and (2) in Figure 4 give the transitional semantics of the restriction operator. Processes in a distributed program may also be composed by a composition operator II similar to that used in CSP [Hoare85]. $P \| Q$ is the parallel composition of processes $P$ and $Q$ with synchronisation of the actions common to both of their alphabets and interleaving of the others. The alphabet of $P \| Q$ is given by the union of their individual alphabets (i.e. $\alpha P \cup \alpha Q$ ).
1a. $\frac{P \stackrel{a}{\longrightarrow} P^{\prime}}{P \uparrow L \stackrel{a}{\longrightarrow} P \uparrow L}\left(a \in L, P^{\prime} \neq \Pi\right)$
2a. $\frac{P \stackrel{a}{\longrightarrow} P^{\prime}}{P \uparrow L \stackrel{\tau}{\longrightarrow} P \uparrow L}\left(a \notin L, P^{\prime} \neq \Pi\right)$
1b. $\frac{P \stackrel{a}{\longrightarrow} \Pi \Pi}{P \uparrow L \stackrel{a}{\longrightarrow} \Pi}(a \in L)$
2b. $\frac{P \stackrel{a}{\longrightarrow} \Pi \Pi}{P \uparrow L \stackrel{\tau}{\longrightarrow} \Pi}(a \notin L)$
3a. $\frac{P \stackrel{a}{\longrightarrow} P^{\prime}}{P\left\|\stackrel{a}{\longrightarrow} P^{\prime}\right\| Q}\left(a \notin \alpha Q, P^{\prime} \neq \Pi\right)$
4a. $\frac{Q \stackrel{a}{\longrightarrow} Q^{\prime}}{P\|Q \stackrel{a}{\longrightarrow} P\| Q^{\prime}}\left(a \notin \alpha P, Q^{\prime} \neq \Pi\right)$
3b. $\frac{P \stackrel{a}{\longrightarrow} \Pi}{P \| Q \stackrel{a}{\longrightarrow} \Pi}(a \notin \alpha Q)$
4b. $\frac{Q \stackrel{a}{\longrightarrow} \Pi \Pi}{P \| Q \stackrel{a}{\longrightarrow} \Pi}(a \notin \alpha P)$

5a. $\frac{P \stackrel{a}{\longrightarrow} P^{\prime} Q \stackrel{a}{\longrightarrow} Q^{\prime}}{P\left\|Q \stackrel{a}{\longrightarrow} P^{\prime}\right\| Q^{\prime}}\left(a \in \alpha P \cap \alpha Q, P^{\prime} \neq \Pi, Q^{\prime} \neq \Pi\right)$

5b. $\frac{P \stackrel{a}{\longrightarrow} P^{\prime} Q \stackrel{a}{\longrightarrow} Q^{\prime}}{P \| Q \stackrel{a}{\longrightarrow} \Pi}\left(a \in \alpha P \cap \alpha Q, P^{\prime}=\Pi \vee Q^{\prime}=\Pi\right)$

Figure 4: Rules of the Restriction and Composition Operators 

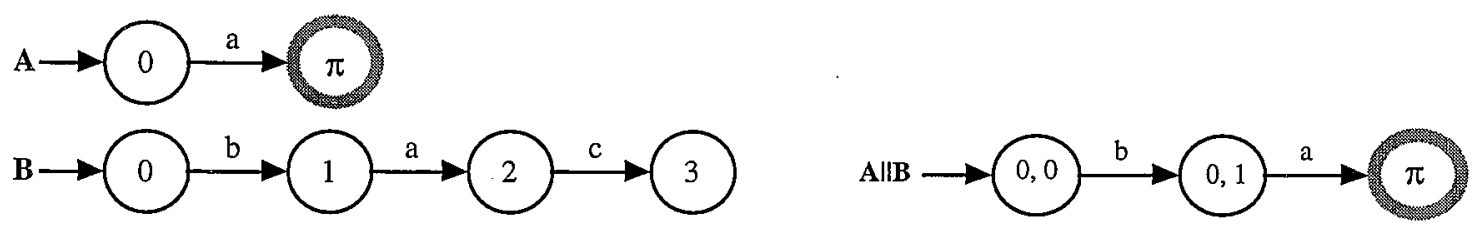

Figure 5: (a) Processes $A, B$ and (b) their Composite Process $A \| B$

Rules (3), (4) and (5) in Figure 4 give the transitional semantics of the composition operator. The operator is both commutative and associative. Given two processes $A$ and $B$ behaving as described in Figure 5(a), we can construct a composite process $A \| B$ behaves as Figure 5(b) by applying the rules. Here, the alphabet of $A$ and $B$ is $\{a\}$ and $\{a, b, c\}$, respectively. States in the composite process are given in tuples where the first and second fields refer to the state in processes $A$ and $B$, respectively. The rules also require that a composite process be trapped at state $\pi$ if any of its constituent processes is trapped. For simplicity, states in a composite process will be given in tuples only if explanation requires relating them to the states in the constituent processes; otherwise they will be simply labelled using integers. For example, states in Figure 5(b) are labelled by integers 0 and 1 instead of tuples $(0,0)$ and $(0,1)$ if the discussion of $\mathrm{AllB}$ does not need to refer to the original states in $\mathrm{A}$ and $\mathrm{B}$.

\subsection{Behavioural Equivalences}

Informally, the notion of observation on a process is postulated to describe the process behaviour perceivable by an observer performing experiments on the process. Behaviour equivalence is a concept to identify a pair of processes which cannot be distinguished by such observations. Two processes are said to be in weak equivalence (denoted as $\approx$ ) when their observable behaviours are indistinguishable. When the behaviours of these two processes are indistinguishable even when taking into account their unobservable behaviours, they are said to be in strong equivalence (denoted as ). Naturally, strong equivalence is a subset of weak equivalence. Comprehensive treatment of strong and weak equivalences can be found in the work of Milner [Milner89b]. Formally, let $\wp$ and $\Sigma$ be the universal set of processes and that of actions including $\tau$, respectively. Formally, strong equivalence $\sim$ is the union of all relations $\boldsymbol{R} \subseteq \wp \times \wp$ satisfying that $(P, Q) \in \boldsymbol{R}$ implies:

(i) $\alpha P=\alpha Q$;

(ii) for all $a \in \Sigma$ :

(a) $P \stackrel{a}{\longrightarrow} P^{\prime}$ implies $\exists Q^{\prime}, Q \stackrel{a}{\longrightarrow} Q^{\prime}$ and $\left(P^{\prime}, Q^{\prime}\right) \in \boldsymbol{R}$.

(b) $Q \stackrel{a}{\longrightarrow} Q^{\prime}$ implies $\exists P^{\prime}, P \stackrel{a}{\longrightarrow} P^{\prime}$ and $\left(P^{\prime}, Q^{\prime}\right) \in \boldsymbol{R}$. 
Let $P \stackrel{a}{\Rightarrow} P^{\prime}$ denote $P(\stackrel{\tau}{\longrightarrow}) * P^{\prime}$ if $a=\tau$; otherwise $P(\stackrel{\tau}{\longrightarrow})^{*} \rightrightarrows(\stackrel{\tau}{\longrightarrow}) * P^{\prime}$. Weak equivalence $\approx$ is the union of all relations $\boldsymbol{R} \subseteq \wp \times \wp$ satisfying that $(P, Q) \in \boldsymbol{R}$ implies:

(i) $\quad \alpha P=\alpha Q$;

(ii) for all $a \in \Sigma$ :

(a) $P \stackrel{a}{\longrightarrow} P^{\prime}$ implies $\exists Q^{\prime}, Q \stackrel{a}{\Rightarrow} Q^{\prime}$ and $\left(P^{\prime}, Q^{\prime}\right) \in \boldsymbol{R}$.

(b) $Q \stackrel{a}{\longrightarrow} Q^{\prime}$ implies $\exists P^{\prime}, P \stackrel{a}{\Rightarrow} P^{\prime}$ and $\left(P^{\prime}, Q^{\prime}\right) \in \boldsymbol{R}$.

The key mechanism of compositional reachability analysis is to minimise the number of states of a composite process after action restriction. The minimisation respects the weak equivalence. A minimisation algorithm respecting the weak equivalence can be found in the work by Holzmann [Holzmann91], and Paige [Paige87]. To facilitate our discussion, let us denote $P \Uparrow L$ to be the minimised process derived from $P \uparrow L$.

\subsection{Assumptions}

In the discussion of compositional reachability analysis, we assume that each subsystem hides at its boundary only those internal actions that neither participate in further interprocess communications nor are globally observable. Suppose a program contains primitive processes $P_{1}, \ldots, P_{n}$. Under this assumption, the global state machine constructed by CRA is $\left(P_{I} \| \ldots P_{n}\right) \Uparrow L$, where $L$ is the set of actions that appear in the global state machine. Safety properties are concerned with the absence of undesirable states in a program. In this paper, we confine the state space of a program to the cartesian product of LTSs. We argue that safety properties can be adequately expressed using finite state automata if all primitive processes can be expressed as finite state deterministic machines. The state space formed by the cartesian product of these primitive processes is both finite and deterministic. As such, all undesirable states are uniquely identified by distinct traces that can be expressed using regular expressions and hence finite state automata. 


\subsection{A Gas Station Example}

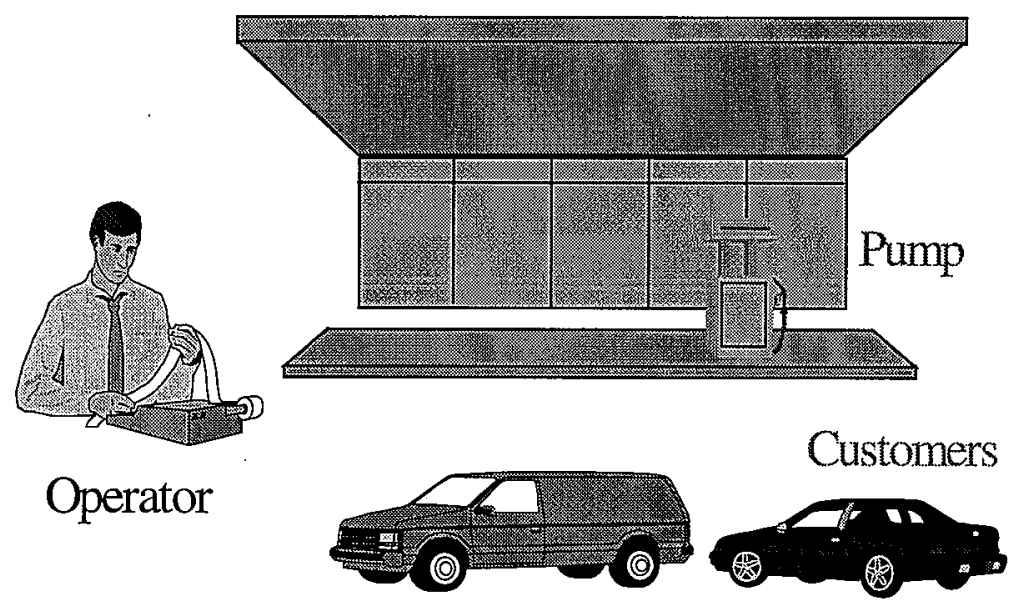

Figure 6: A Gas Station System

As an illustration for our discussion, we present a gas station example (Figure 6) originally proposed by Helmbold and Luckham [Helmbold85]. The example models an automated gas station with an operator, a pump, two customers and a queue holding customers' requests ${ }^{2}$. The design architecture in Figure 7 describes the subsystem structure, constituent processes and interactions for the gas station system. It is specified in an Architecture Description Language (ADL), Darwin ${ }^{3}$ [Magee95], which has a graphical representation as shown in the figure. Interaction between processes is achieved by communication on bindings between matching pairs of service requirements (white dots) and provisions (black dots). Subsystems are introduced to encapsulate internal substructure and interaction. The approach is designed to support modular and comprehensible system design. Decomposition of subsystems terminates at the primitive processes that are represented as shaded rectangles. For analysis, behaviours of primitive processes are given as LTSs; for implementation, primitive processes are realised in some programming language, such as $\mathrm{C}++$.

2 The example can be extended to accommodate more operators, customers and pumps.

${ }^{3}$ Darwin supports the definition of parameterised component types and generic architectures. For analysis and program construction, a generic architecture must be instantiated, evaluating the parameters, elaborating the component definitions and performing interface bindings to generate a particular architectural instance. This can then be analysed or implemented in Regis [Magee94], a distributed programming environment which supports Darwin design specifications. For simplicity, we neglect many of the naming and elaboration issues. 


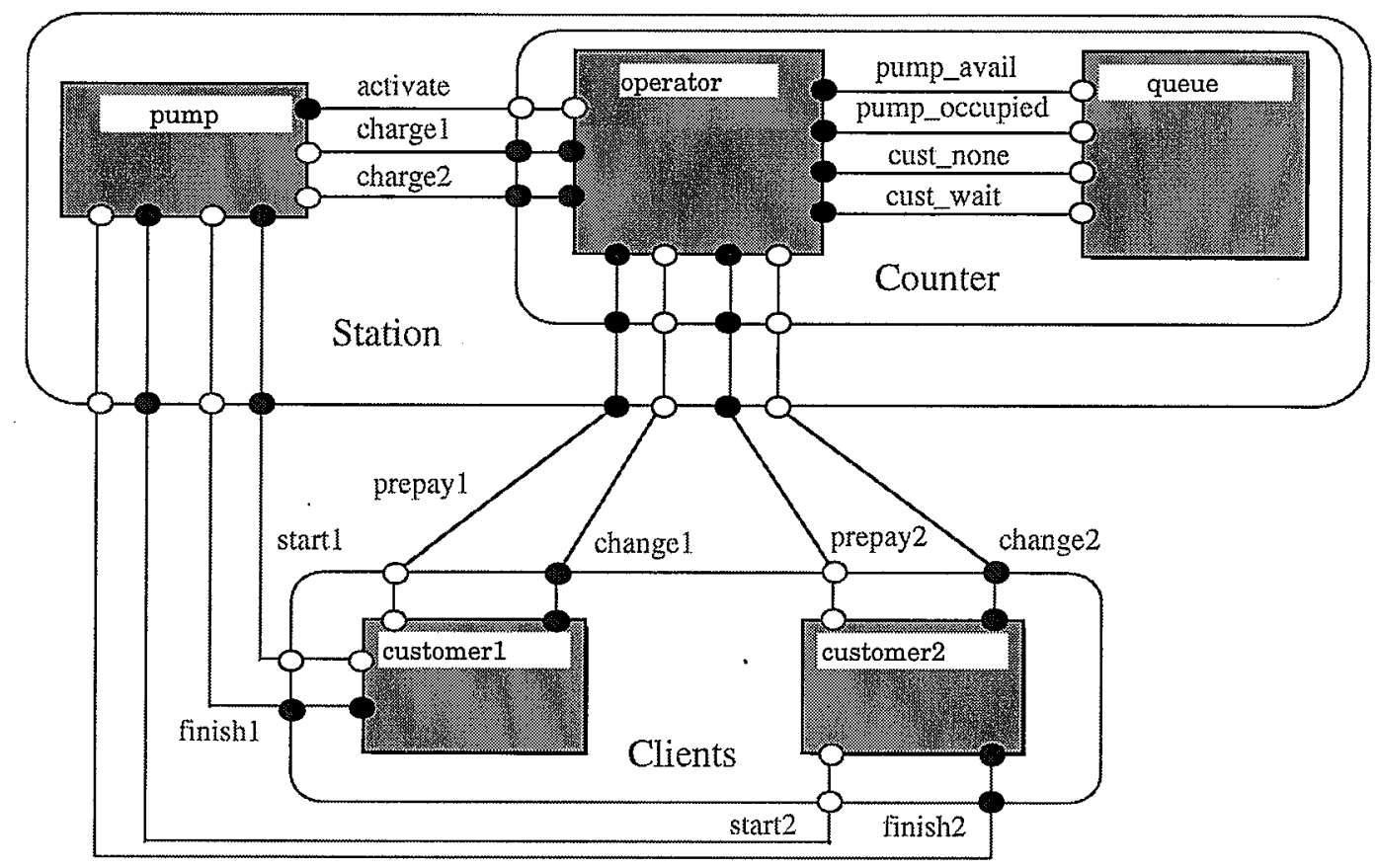

Figure 7: The Design Architecture of the Gas Station System

The design architecture of the gas station postulates a hierarchy of subsystems as shown in Figure 8 . The set of actions exported by a subsystem for interaction is annotated using the restriction operator $\uparrow$. In the hierarchy, a subsystem is a composite process (represented as a box with rounded corners) formed by the parallel composition of its descendent processes, which in turn can either be subsystems or primitive processes (represented as rectangles). For instance, the design architecture specifies that subsystem Counter is composed of processes Operator and Queue, and Station by Counter and Pump. Textually, we write

$$
\begin{array}{r}
\text { Counter }=(\text { Operator } \| \text { Queue }) \Uparrow\{\text { activate, } \text {, chargel }, \text { charge } 2, \\
\text { prepay } 1, \text { prepay } 2, \text { change } 1, \text { change } 2\}
\end{array}
$$

and

Station $=($ Counter $\|$ Operator $) \Uparrow\{$ start1, start 2, finish 1, finish 2, prepay1, prepay2, change1, change2\}. 


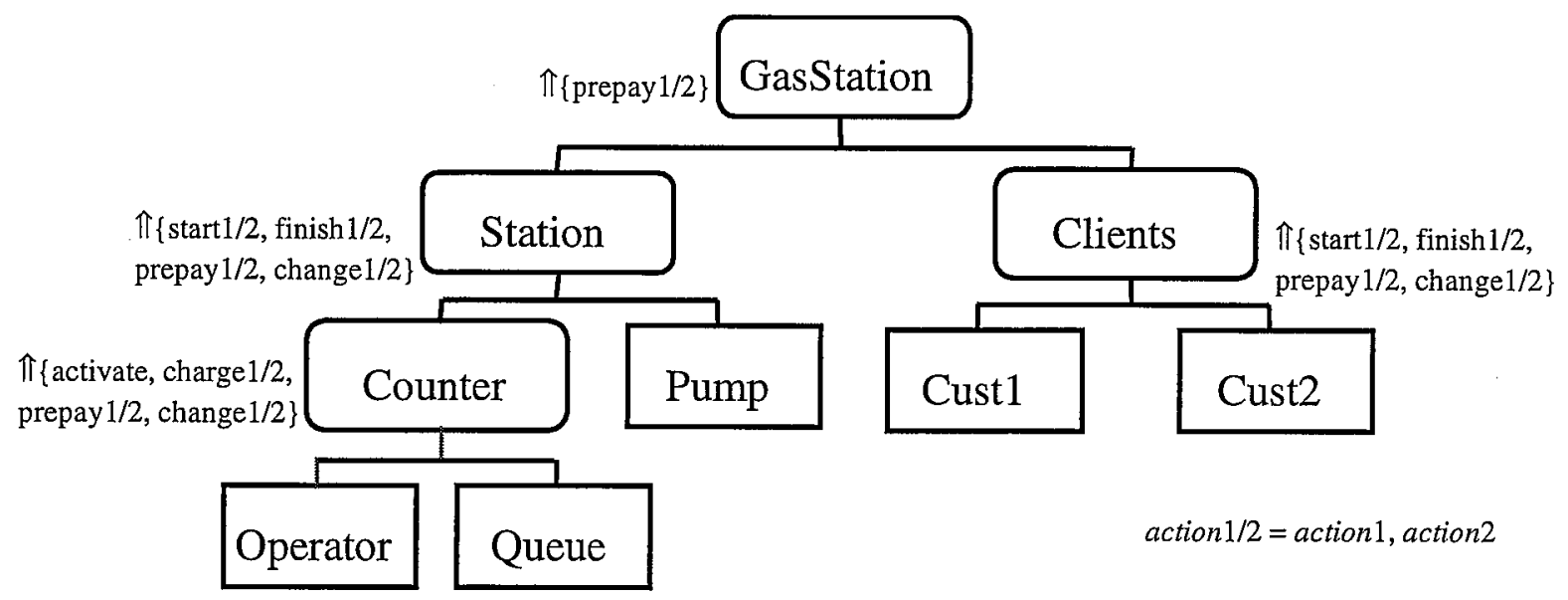

Figure 8: Subsystem hierarchy of the Gas Station System

Figure 9 gives the LTSs presenting the behaviour of the two primitive processes operator and the request queue. The operator may initially choose to accept money prepaid by customers $\left(\right.$ prepay $\left._{i}\right)$ or accept the amount to be charged from the pump (charge $)_{i}$. After accepting money from a customer, the operator activates the pump if it is available; otherwise does nothing if it is occupied. On receiving the charge information from the pump, the operator gives the change (change ${ }_{i}$ ) to the customer and activates the pump again if there are customers waiting for the pump.
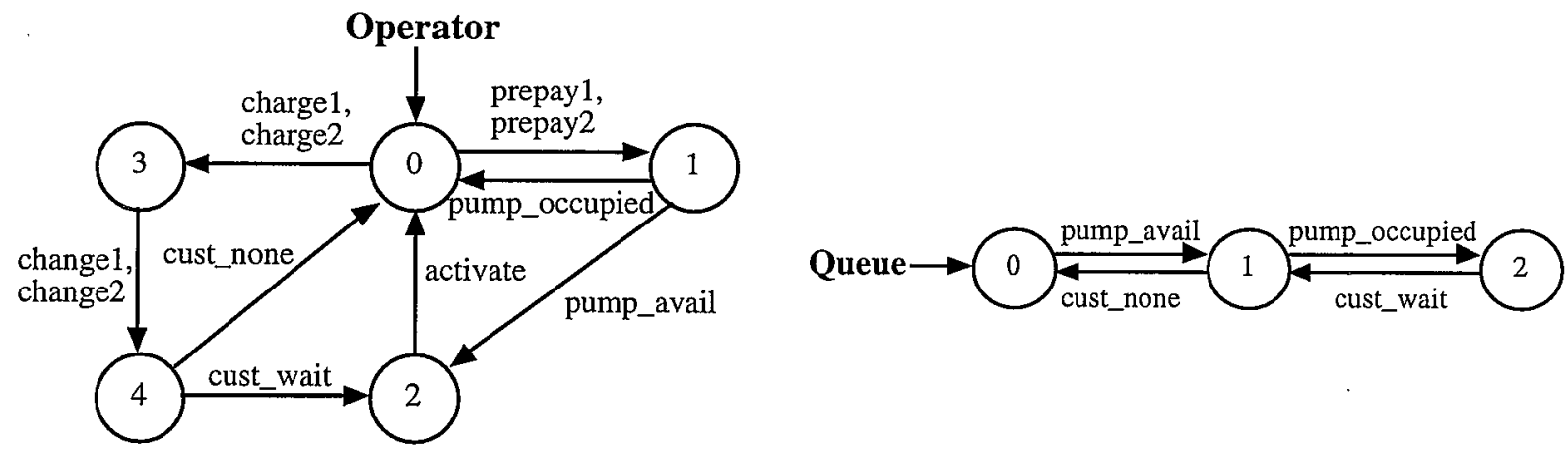

Figure 9: Behaviour of the Operator and the Queue holding customers' requests.

Figure 10 shows the behaviour of the pump and that of the two customers. A customer who has paid the money can start the pump once it has been activated. After starting the pump, the customer may at any time request the pump to finish pumping ( inish $_{i}$ ) and wait for the change from the operator. Upon receiving the "finish" request, the pump informs the operator of the charge information. 

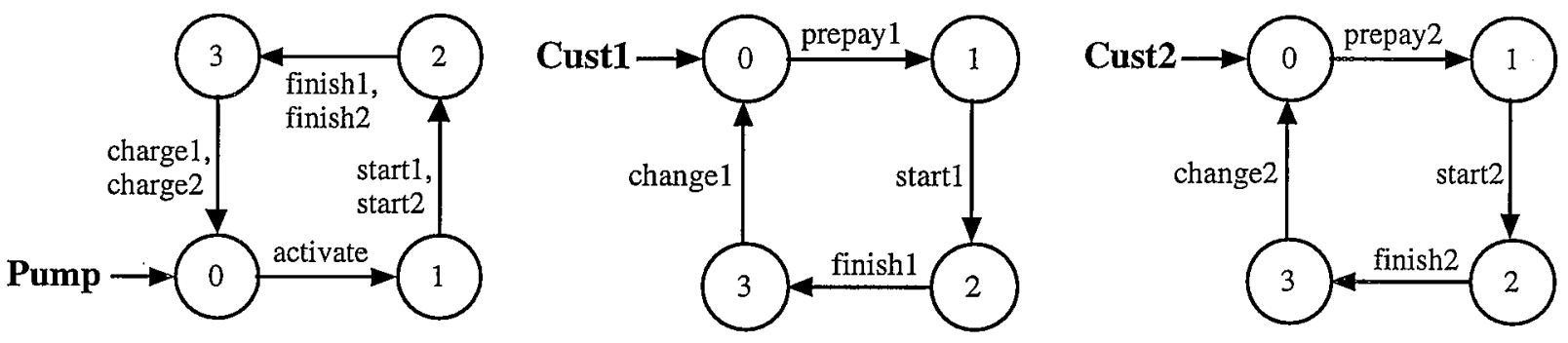

Figure 10: Behaviour of the Pump and two customers Cust1 and Cust2

As mentioned, hiding internal interactions helps make the design and analysis more comprehensible. In the following discussion, let us assume that the software developers want to know if customers can be served in any order, and hence study the system behaviour with respect to actions prepayl and prepay2. In other words, only these two actions are retained as observable in the global LTS of GasStation.

GasStation $=($ Station $\|$ Clients $) \Uparrow\{$ prepay 1, prepay 2$\}$

\section{Enhanced Compositional Reachability Analysis}

\subsection{Limitations of Compositional Reachability Analysis}

As mentioned, compositional derivation of the overall system behaviour has been shown to be a very promising approach for reachability analysis [Sabnani89, Tai93a, Yeh91]. In such compositional reachability analysis (CRA) techniques, the model of the target system is given as an LTS that describes the overall system behaviour. Given a subsystem hierarchy, the LTS of a system is composed step by step from those of its subsystems in a bottom-up manner. In each intermediate step, the LTS of a subsystem is minimised by hiding all internal actions. The For instance, the LTS of GasStation in Figure 8 can be composed in four steps. First, compose the LTS of Counter from Operator and Queue. Second, compose the resultant LTS of Counter with Pump to generate the LTS of Station. Third, construct the LTS of Clients from Cust1 and Cust2. Finally, compose the LTS of GasStation from that of Station and that of Clients. This mechanism of "intermediate minimisation during composition" is attractive for the analysis of modular systems. Figure 11 shows that there is no particular ordering relation between the occurrences of prepayl and prepay 2.

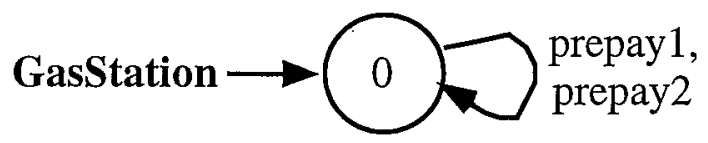

Figure 11: A Global LTS of GasStation 
The key to the success of CRA techniques is to employ a modular software architecture and hide as many internal actions as possible in each subsystem. A subsystem containing fewer observable actions can generally be represented by a simpler LTS. However, the properties that are then available for reasoning in the analysis is constrained by the set of remaining globally observable actions. For instance, the properties that are available for reasoning in the analysis of the GasStation can only be formed by actions prepay1 and prepay2. Safety properties that involve other actions cannot be examined from the global LTS of the GasStation in Figure 11. Examples of these other properties are that the Operator must give the right change to the right customer (Figure 12(a)) and the Pump must complete the service to a customer before serving the other (Figure 12(b)). The former property refers to the expected behaviour of Operator and the latter to that of Pump. If these properties are to be verified in the CRA, actions charge1, charge2, change1, change2, start1, start2, finish1 and finish2 need to be made globally observable. However, this would offend against the hiding principle of CRA techniques and thus undermine the effectiveness of the associated analysis. In the following, we introduce a technique that is capable of checking these safety properties without increasing the set of globally observable actions in the GasStation.

\subsection{Validation of Safety Properties}

A safety property prescribes the set of legitimate traces. This can be represented by a deterministic property automaton $T=(S, A, \Delta, q)$, which is free of state $\pi$ and internal action $\tau$ (see Figure 12). A property is said to be violated, within the context of a distributed program, when some processes can perform a trace not acceptable to the property automaton. The detection mechanism is required to make the violation, if any, globally observable. To achieve this, we first derive an LTS referred to as an image automaton based on a given property automaton. The image automaton traps possible violation at the $\pi$ state. This image automaton is then composed with the processes to which the automaton applies. As the $\pi$ state is preserved by both the restriction operator and the composition operator, possible violation is made globally observable. Violation of safety properties can therefore be checked by examining the existence of the $\pi$ state in the global LTS.

An image automaton $T^{\prime}=\left(S \cup\{\pi\}, A, \Delta^{\prime}, q\right)$ can be derived from a given property automaton $T$ $=(S, A, \Delta, q)$ by defining $\Delta^{\prime}$ to be $\Delta \cup\left\{(s, a, \pi) I(s, a) \in S \times A \wedge \nexists s^{\prime} \in S:\left(s, a, s^{\prime}\right) \in \Delta\right\}$. 
(a)

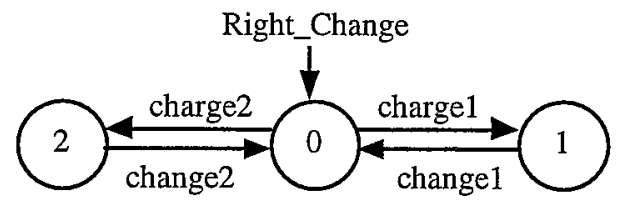

(b)

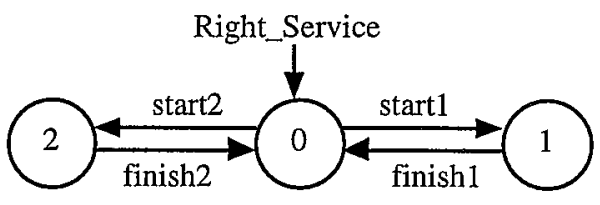

Figure 12: Two Property Automata Specified by Users
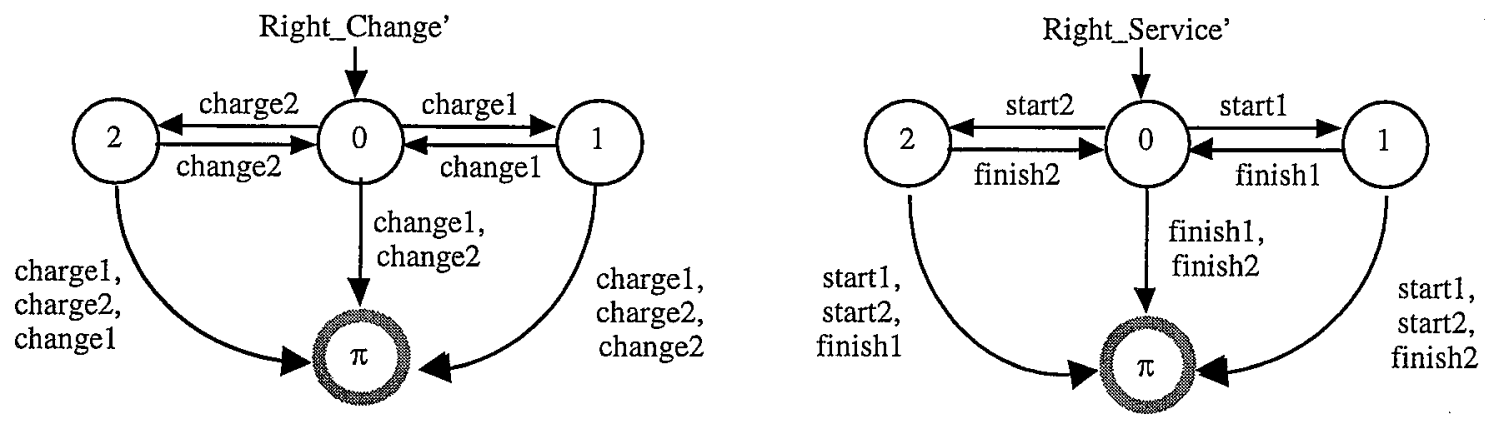

Figure 13: Image Automata of (a) Right_Change and (b) Right_Service

For example, Figure 13 gives the corresponding image automata derived from Right_Change and Right_Service of Figure 12. The image automaton so constructed satisfies two conditions:

(i) $T$ and $T^{\prime}$ have the same set of nontrapping traces (i.e. $\operatorname{tr}(T)$ ); and

(ii) for any process $P, P \| T^{\prime}$ does not contain trapping traces iff $\operatorname{tr}(P \uparrow \alpha T) \subseteq \operatorname{tr}(T)$.

\section{Proof of condition (i)}

Step (1) in the construction of image automaton ensures that $\Delta$ is a subset of $\Delta^{\prime}$. Step (2) ensures that for any transition $\left(s, a, s^{\prime}\right)$ belongs to $\Delta^{\prime}-\Delta, s^{\prime}$ equals $\pi$. Hence, the $\Delta^{\prime}$ and $\Delta$ contain the same set of transitions that do not involve state $\pi$; i.e.

$$
\left\{\left(s, a, s^{\prime}\right) \mid\left(s, a, s^{\prime}\right) \in \Delta^{\prime} \wedge s^{\prime} \neq \pi\right\}=\left\{\left(s, a, s^{\prime}\right) \mid\left(s, a, s^{\prime}\right) \in \Delta \wedge s^{\prime} \neq \pi\right\} .
$$

Since $T$ and $T^{\prime}$ share the same initial state $q$, all nontrapping traces that can be performed by $T$ can also be performed by $T^{\prime}$ and vice versa. As a result, $T$ and $T^{\prime}$ have the same set of nontrapping traces, which is equal to $\operatorname{tr}(T)$.

\section{Proof of condition (ii)}

Case (1): if part: Assume $\operatorname{tr}(P \uparrow \alpha T) \subseteq \operatorname{tr}(T) . \operatorname{tr}((P \| T) \uparrow \alpha T)$ equals $\{t \mid t \in \operatorname{tr}(P \uparrow \alpha T) \cap \operatorname{tr}(T)\}$ which in turn equals $\{t \mid t \in \operatorname{tr}(P \uparrow \alpha T) \cap \operatorname{tr}(T)\} \cup\{t \mid t \in \operatorname{tr}(P \uparrow \alpha T) \cap(\operatorname{tr}(T)-\operatorname{tr}(T))\}$. The image process $T^{\prime}$ is so constructed that only those traces in $\operatorname{tr}\left(T^{\prime}\right)-\operatorname{tr}(T)$ can lead $T^{\prime}$ in a trap state. Hence, $\{t \mid t \in \operatorname{tr}(P \uparrow \alpha T) \cap \operatorname{tr}(T)\}$ and $\{t \mid t \in$ $\left.\operatorname{tr}(P \uparrow \alpha T) \cap\left(\operatorname{tr}\left(T^{\prime}\right)-\operatorname{tr}(T)\right)\right\}$ represent the set of nontrapping and trapping traces respectively in $(P \| T) \uparrow \alpha T$. As $\operatorname{tr}\left(P \uparrow \alpha T \subseteq \operatorname{tr}(T), \operatorname{tr}(P \uparrow \alpha T) \cap\left(\operatorname{tr}\left(T^{\prime}\right)-\operatorname{tr}(T)\right)\right.$ is an empty set. As a result, there are no trapping traces in $(P$ $\| T) \uparrow \alpha T,(P \| T) \uparrow \alpha T$ is nontrappable. Thus, $P \| T$ ' is also nontrappable.

Case (2): only-if part: Assume $P \| T^{\prime}$ is nontrappable. Let us suppose $\operatorname{tr}(P \uparrow \alpha T) \nsubseteq \operatorname{tr}(T)$. This implies that $P \uparrow \alpha T$ can perform a trace $t$ that does not belong to $\operatorname{tr}(T)$. We note that any prefix of $t$ can be performed by $P \uparrow \alpha T$. Let $s$ be a prefix of $t$ such that $T$ can perform all prefixes of $s$ but not the $s$ itself. By the method we 
construct the image process, $s$ can also be performed by $T^{\prime}$. As a result, $s$ is a trapping trace in $T^{\prime}$. Thus $s$ is a trapping trace in $(P \uparrow \alpha T \| T)$, which is equal to $(P \| T) \uparrow \alpha T$. By the semantics of restriction operator, the existence of a trapping trace in $(P \| T) \uparrow \alpha T$ implies the existence of a trapping trace in $P \| T^{\prime}$. This contradicts to the assumption that $P \| T^{\prime}$ is nontrappable. Thus, the supposition cannot hold.

Condition (ii) enables us to detect violation of safety properties in a system by checking the existence of trapping traces in the composite process formed by the system and the image automata. If trapping traces exist in the composite process, some safety properties are violated.

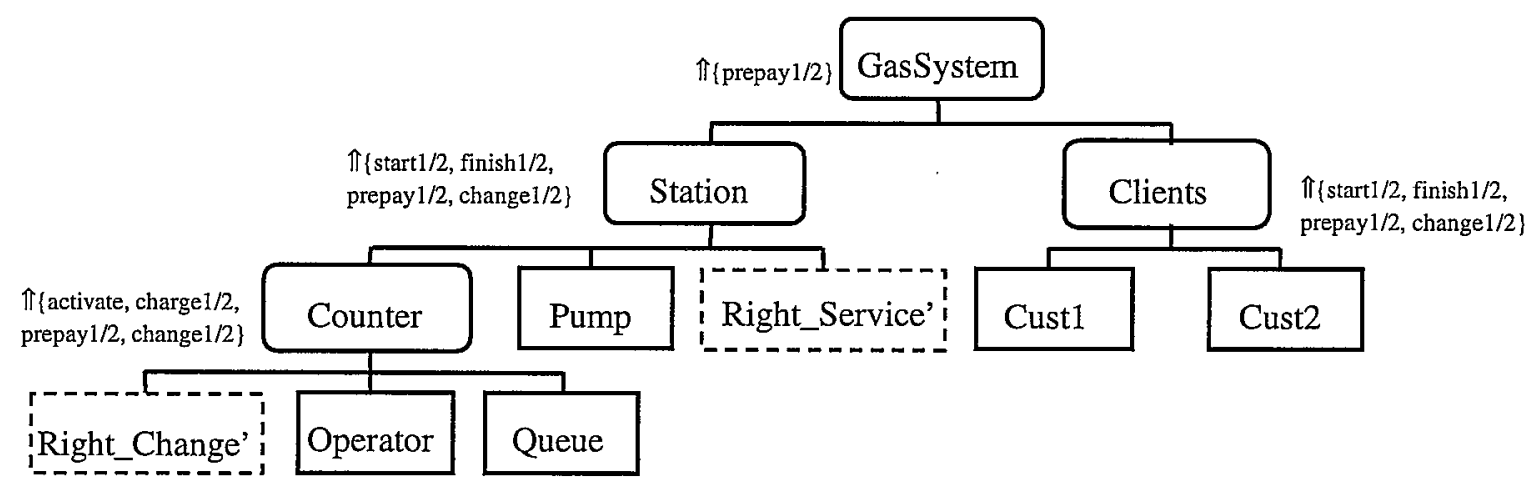

Figure 14: The subsystem hierarchy for checking safety properties Right_Change' and Right_Service' which are associated with the Operator and Pump respectively

In CRA, an image automaton can be composed directly with a constituent process whose alphabet is a superset of that of the automaton. For example, Figure 14 shows the modified subsystem hierarchy to include the image automata of Figure 13. As shown in the modified hierarchy, Right_Change' and Right_Service' are composed (in parallel) respectively with the processes Operator and Pump to which these properties refer. An image automaton is placed within the subsystem whose property is to be examined. For instance, the image automaton Right_Change' is placed within Counter as it concerns the actions \{charge1, charge2, change1, change2 $\}$ in which the components of Counter participate. Figure 15 gives the global LTS derived by the CRA based on the hierarchy in Figure 14. Since the global LTS contains trapping traces, the Gas Station system does not satisfy both safety properties represented by the property automata Right_Change and Right_Service.

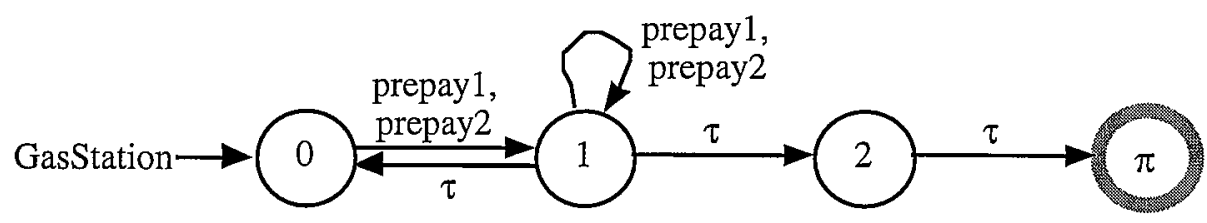

Figure 15: The global LTS derived by the CRA based on the hierarchy in Figure 14 


\section{Debugging}

\subsection{Locating the Violation}

The above technique indicates whether all specified safety properties are satisfied. However, users would normally wish to know which particular safety properties are violated, and in what way. To provide this information, the CRA technique needs to be further enhanced with a mechanism to keep track of the relation between those transitions leading to the trap state $\pi$ in the global LTS and those in the image automata.

Let $P$ be a process $\langle S, A, \Delta, q\rangle$, and $\mu_{P}$ be a mapping $((S-\{\pi\}) \times A) \rightarrow 2((\mathcal{S}-(\pi)) \times \Sigma \times(\pi))$, where $\mathfrak{J}$ and $\Sigma$ denote the universal sets of states and actions, respectively. The value of $\mu_{P}(s, a)$ indicates the set of trapping transitions in the image automata that contribute to a trapping transition $(s, a, \pi)$ in $P$. Let $\vartheta_{s}$ denote the process $\langle S, A, \Delta, s\rangle$, where $s$ is a reachable state in $P$. The mapping $\mu_{P}$ is defined as follows:

(i) if $P$ is an image automaton, $\mu_{P}(s, a)=\{(s, a, \pi)\}$ if $(s, a, \pi)$ exists in $P$; otherwise $\varnothing$;

(ii) $\quad$ if $P=Q \| R, \mu_{P}\left(\left(s_{Q}, s_{R}\right), a\right)=\mu_{Q}\left(s_{Q}, a\right) \cup \mu_{R}\left(s_{R}, a\right)$;

(iii) if $P \approx Q \uparrow L, \mu_{P}(s, a)=\left\{v \mid v \in \mu_{Q}\left(s^{\prime}, a\right)\right.$ such that $\left.\vartheta_{s} \approx \vartheta_{s^{\prime}} \uparrow L\right\}$.

Since the set union operation can be computed using bitwise arithmetic in imperative programming languages, its computational complexity is linear in time and space. The computation of $\mu$ does not increase the computational complexity of the error detection mechanism.

Using the above definition, $\mu_{\text {Right_Change }}\left(0_{\text {Right_Change' }}\right.$, chargel $)$ and $\mu_{\text {Right_Change }}\left(0_{\text {Right_Change' }}\right.$, changel $)$ are defined to be $\varnothing$ and $\left\{\left(0_{\text {Right_Change' }^{\prime}}\right.\right.$ change $\left.\left.1, \pi\right)\right\}$, respectively. Here, $0_{\text {Right_Change' }}$ represents the state 0 in the image automaton Right_Change'. Applying the definition to processes constructed by CRA, $\mu_{\text {Gasstation }}\left(2_{\text {Gasstation, }} \tau\right)$ takes a value of $\left\{\left(1_{\text {Right_Change' }}\right.\right.$, change $\left.2, \pi\right), \quad\left(2_{\text {Right_Change' }}\right.$, change1,$\left.\left.\pi\right)\right\}$. The global LTS of GasStation constructed based on the hierarchy of Figure 14 is given in Figure 15. It contains only one trapping transition $\left(2_{\text {Gasstation }}, \tau, \pi\right)$. The value of $\mu_{\text {GasStation }}\left(2_{\text {Gasstation }}, \tau\right)$ suggests that the safety violation occurs at transitions $\left(1_{\text {Right_change' }}\right.$, change $\left.2, \pi\right)$ and $\left(2_{\text {Right_Change' }}\right.$, change $\left.1, \pi\right)$ of the image automata Right_Change' (Figure 13a). The former represents a situation where charge 1 can be followed by change 2 and the latter represents a situation where charge 2 can be followed by change1. In both situations, customers receive the wrong change. On the other hand, the safety property specified by property automaton Right_Service (Figure 12b) is not violated by the GasStation. 


\subsection{Construction of Debugging Traces}

A common error reporting technique in behavioural analysis is to present a debugging trace that leads the system being analysed to a trap state. Users may then follow the given trace to gain a deeper understanding of reported errors. While analysis of large systems is better done incrementally with details suppressed at intermediate stages, information provided by debugging traces should be as detailed as possible. A debugging trace should show explicitly how each primitive process of a system reaches a state where a desirable property is violated. Thus, a debugging trace of the GasStation system may include unobservable actions performed by Station, Clients, Pump and Counter. An example leading to the violation of Right_Change is 〈prepayl, pump_avail, activate, start1, finishl, charge2, changel〉. Unobservable actions in a trace can be recovered using the following reconstruction algorithm proposed by Yeh and Young [Yeh93b]. The algorithm (Figure 16) assumes the availability of a global trace routine gtrace which, given a list of processes and a trace of observable actions as input, returns a detailed sequence of moves. A move is a (sources, actions) pair containing the action(s) taken in the move and the originating process(es) of the action(s). The algorithm successively reconstructs unobservable actions in a trace according to the subsystem hierarchy deployed for compositional analysis. The predicate primitive checks if a given process is primitive. The routine actions_to_moves converts a list of actions into a list of moves by adding the source information; project extracts actions of a particular process; and compact removes invisible internal actions. The routine reconstruct aggregates individual moves exhibited by each child at a lower level into a single list of moves.

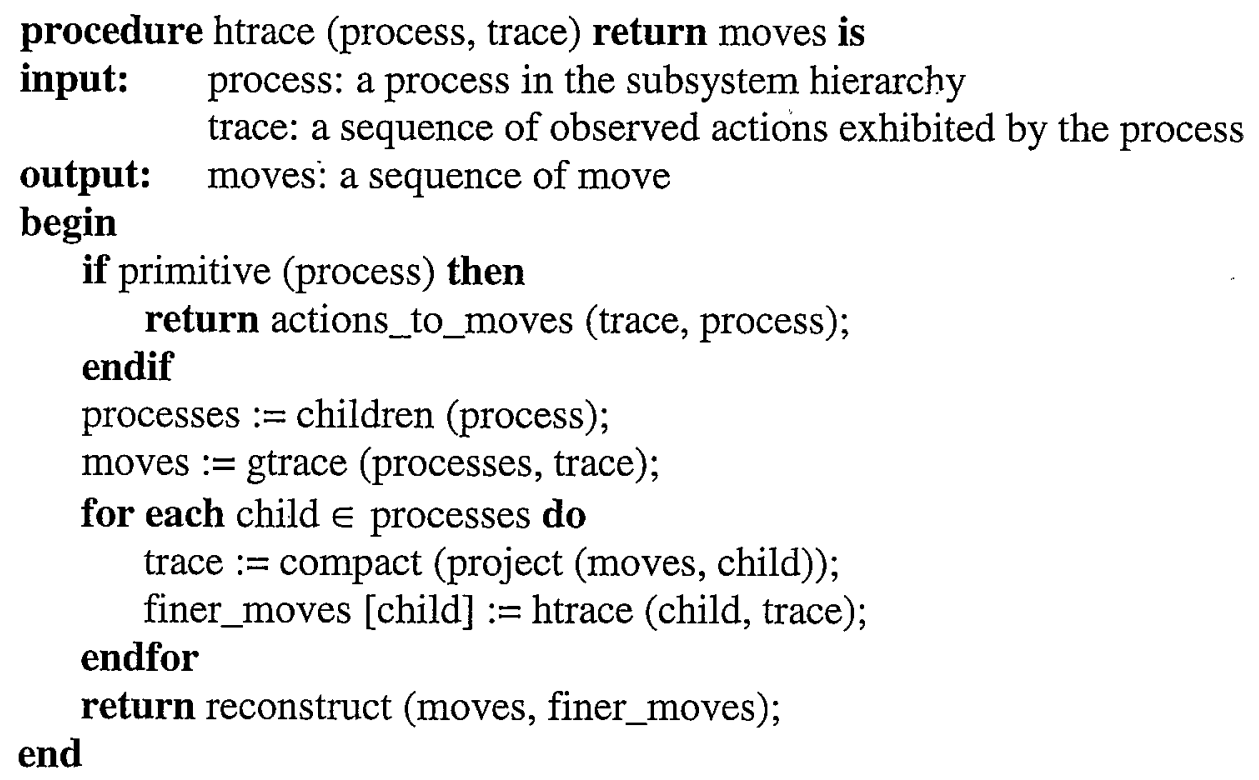

Figure 16: Trace Reconstruction Algorithm 


\subsection{Regression Checking by Re-structuring}

Debugging facilitities of an analysis tool should ideally go beyond the generation of debugging traces. Facilities should be provided to reduce the effort in fixing errors and reanalysis. To fix a reported error, designers may modify the specification of some processes based on the analysis results and their intuition / preferences. For instance, the designer of the Gasstation system may choose to modify the Pump to try and fix the violation of Right_Change. At the same time, the modified system should continue to satisfy the other desirable property Right_Service. To ensure this, the system needs to be checked again each time that it has been modified. We refer this to as regression checking since debugging is likely to be a trial-and-error exercise. For instance, the Pump specification may be modified many times before the error is actually fixed. The effort of regression checking could contribute to a significant part of the software development cost. This effort can, to some extent, be relieved by the use of compositional analysis, where only those subsystems affected by a change need be checked again. For example in Figure 17, only subsystems NO, N1 and N5 need be re-constructed in the subsystem hierarchy when the primitive processes coloured in black are modified. However the effort of regression checking may still be expensive for large systems where modifications are less localised and many subsystems are affected.

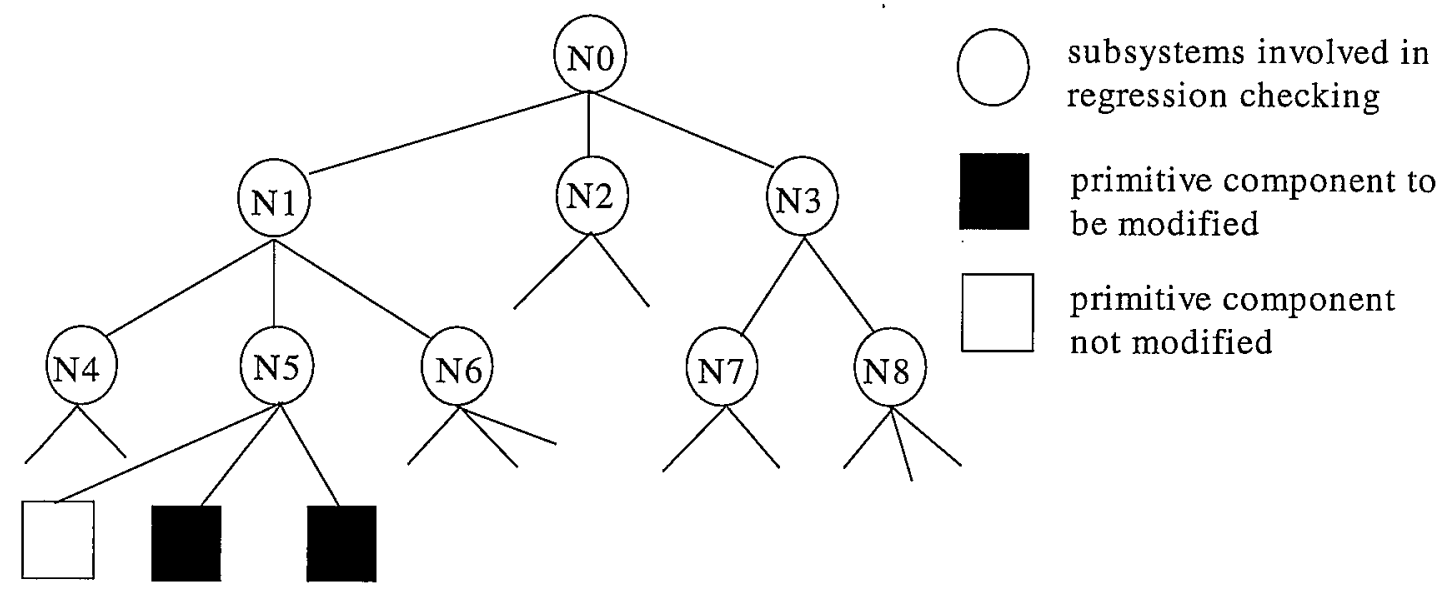

Figure 17: A Subsystem Hierarchy

A more effective approach is to avoid regression checking of subsystems wherever possible. To achieve this, we need to re-structure the subsystem hierarchy and construct a minimised, composite LTS for all processes in the system other than the subsystem being modified. The re-structuring algorithm is presented in Figure 18. 
procedure re-structure $\left((V, E), V_{c}\right)$ return $\left(V^{\prime}, E^{\prime}\right)$ is

input: $(\mathrm{V}, \mathrm{E})$ : a tree representing the original subsystem hierarchy

$V_{c}$ : a set of primitive processes to be modified

output: a tree $\left(V^{\prime}, E^{\prime}\right)$ representing the re-structured subsystem hierarchy

begin

1. $\mathrm{V}^{\prime}:=\mathrm{V} \cup\left\{\mathrm{v}_{0}{ }^{\prime}\right\}$, where $\mathrm{v}_{0}{ }^{\prime} \notin \mathrm{V} ;\left(^{*} \mathrm{v}_{\mathrm{o}}{ }^{\prime}\right.$ is the new root of tree $\left.\left(\mathrm{V}^{\prime}, \mathrm{E}^{\prime}\right)^{*}\right)$

2. $E^{\prime}:=\mathrm{E} \cup\left\{\left(\mathrm{v}_{\mathrm{o}}, \mathrm{v}_{\mathrm{o}}\right)\right\}$;

$\left({ }^{*} \mathrm{v}_{0}\right.$ is the old root of tree $\left.(\mathrm{V}, \mathrm{E}) *\right)$

3. for each $v \in V_{c}$ do

4. $\quad E^{\prime}:=E^{\prime} \cup\left\{\left(v_{0}{ }^{\prime}, v\right)\right\}$;

5. endfor

6. $V_{s}:=\operatorname{sibling}_{(V, E)}\left(V_{c}\right)-V_{c}$;

7. for each $\vee \in V_{S}$ do

8. $\quad E^{\prime}:=E^{\prime} \cup\left\{\left(v_{0}, v\right)\right\} ; \quad$ (* attach siblings to the old root*)

9. endfor

10. $\mathrm{V}_{\mathrm{p}}:=\operatorname{parent}_{(\mathrm{V}, \mathrm{E})}\left(\mathrm{V}_{\mathrm{c}}\right) ; \quad\left(*\right.$ set of immediate parents of vertices in $\left.\mathrm{V}_{\mathrm{c}}{ }^{*}\right)$

11. for each $v \in V_{p}$ do

12. remove $v$ from $\left(V^{\prime}, E^{\prime}\right)$;

13. endfor

end

Figure 18: Re-structuring Algorithm

The re-structure algorithm takes $V_{c}$, the processes to be modified, and a tree $(V, E)$ that records the original subsystem hierarchy, where $V$ and $E$ represent the vertices and edges, respectively. Upon termination, it returns a re-structured hierarchy recorded as $\left(V^{\prime}, E^{\prime}\right)$. Statements 1 and 2 insert a new root $v_{o}^{\prime}$ in $\left(V^{\prime}, E^{\prime}\right)$. The new root $v_{o}^{\prime}$ is then made to be a node that links directly to $v_{o}$ and those modified primitive processes in $V_{c}$. Afterwards, the old root $v_{o}$ is made to be a subsystem that holds all processes in the system other than those in $V_{c}$. The function $\operatorname{sibling}_{(V, E)}\left(V_{c}\right)$ returns a set of processes in $(V, E)$ that share the same immediate parents as those in $V_{c}$. As these sibling processes are placed under the same immediate subsystems with those being modified, they are likely to have close interaction with each other. As such, sibling processes are better placed as near as possible to the modified ones. This is achieved by putting these sibling processes directly under the old root $v_{o}$. The function parent $_{(V, E)}\left(V_{c}\right)$ returns a set of immediate parents of vertices in $V_{c}$. With all immediate successors having been relinked, these parent nodes can be removed from the tree. Note that the algorithm may also be applied to cases where primitive processes are scattered in the subsystem hierarchy and are embedded in more than one immediate subsystem.

\subsection{The Mechanism of Regression Checking}

We need to confirm that the re-structuring process preserves the safety properties of the 
original systems. In the re-structured hierarchy, internal actions that involve only process interactions within a subsystem are hidden at its boundary. Since the re-structured hierarchy is different from the original one, the set of actions restricted by each subsystem may differ before and after re-structuring. Suppose the program behaves as $S$ and $S^{\prime}$ before and after restructuring, respectively. $S^{\prime}$ is weakly equivalent to $P \uparrow L$, where $P$ is the state machine directly composed from the primitive processes of the program and $L^{\prime}$ is the set of actions that appear in $S^{\prime}$. Thus $S^{\prime}$ preserves the safety properties of $P$. As $S$ is again weakly equivalent to $P \uparrow L$ where $L$ is the set of actions appear in $S, S^{\prime}$ preserves the safety properties of $S$.

Using the re-structuring algorithm, the hierarchy in Figure 17 is transformed to that in Figure 19 for regression checking. In the first round of regression checking, the number of subsystems that need to be reconstructed in the re-structured hierarchy is no more than that required in the original hierarchy. The effort of regression checking is significantly reduced in subsequent attempts because only the LTS of the new root $N O^{\prime}$ needs be re-constructed regardless of the tree size and structure. The approach of regression checking using restructuring is particularly useful if the changes affect many subsystems.

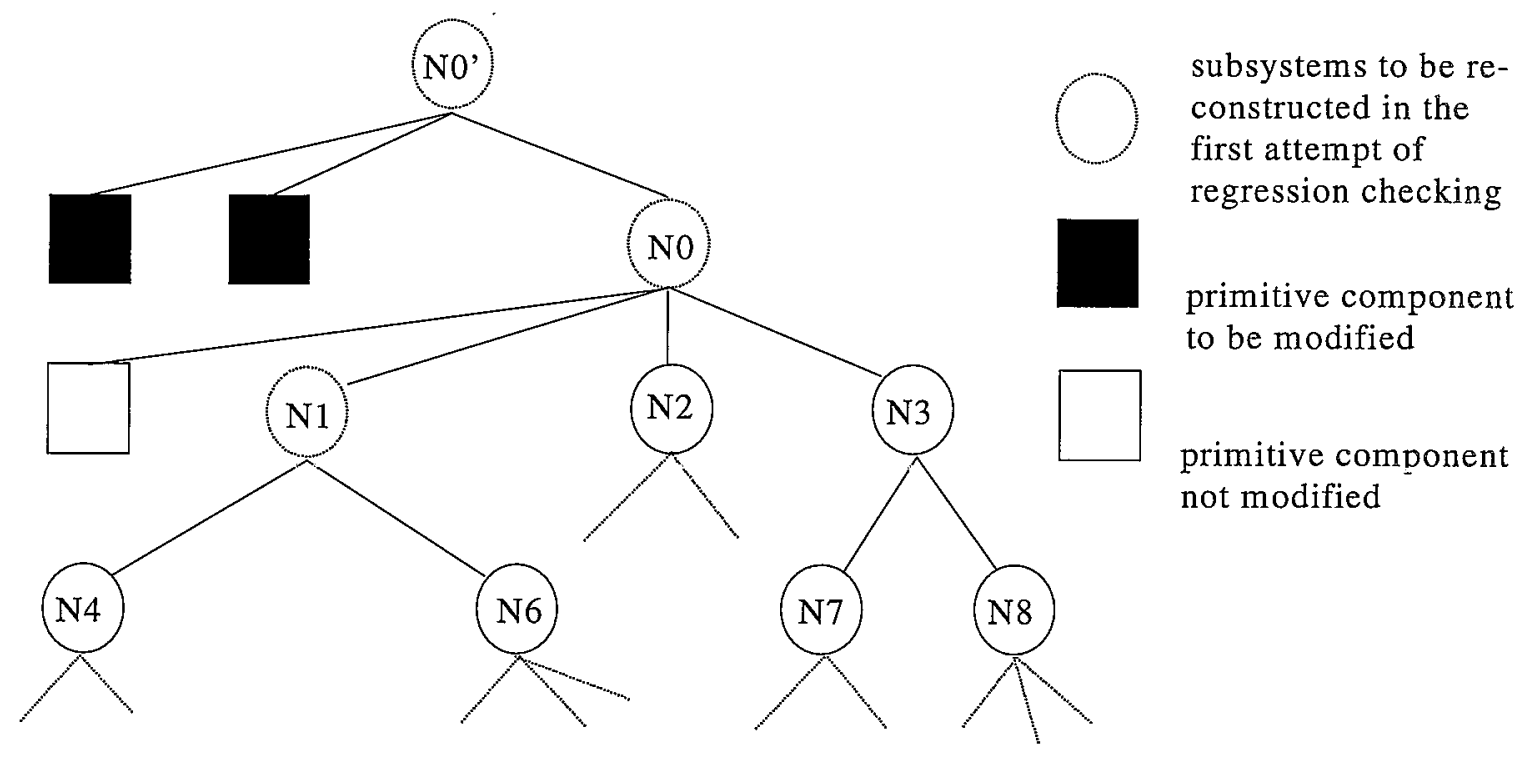

Figure 19: A Re-structured Subsystem Hierarchy

Clearly, this re-structuring approach does not necessarily work if subsystems are constructed using conventional CRA, without the inclusion of trapping states. This is because the composite LTS constructed may only check properties involving actions at the interface of the modified subsystem [Clarke89]. To illustrate the point, let us consider the gas control system as an example and suppose that Pump is the primitive process to be modified. The property Right_Change which involves actions not belonging to the interface of Pump cannot be 
checked using a re-structured hierarchy. As a result, the enhanced CRA technique needs to be employed for regression checking. The inclusion of the $\pi$ state allows the satisfiability conditions of subsystem properties to be propagated up a subsystem hierarchy even though these properties may involve hidden actions. These properties are satisfied if and only if the LTS constructed for the root in the subsystem hierarchy is nontrappable. In the re-structuring algorithm, property automata are treated as primitive processes not to be modified.

\subsection{Regression Checking of the Gas Control System}

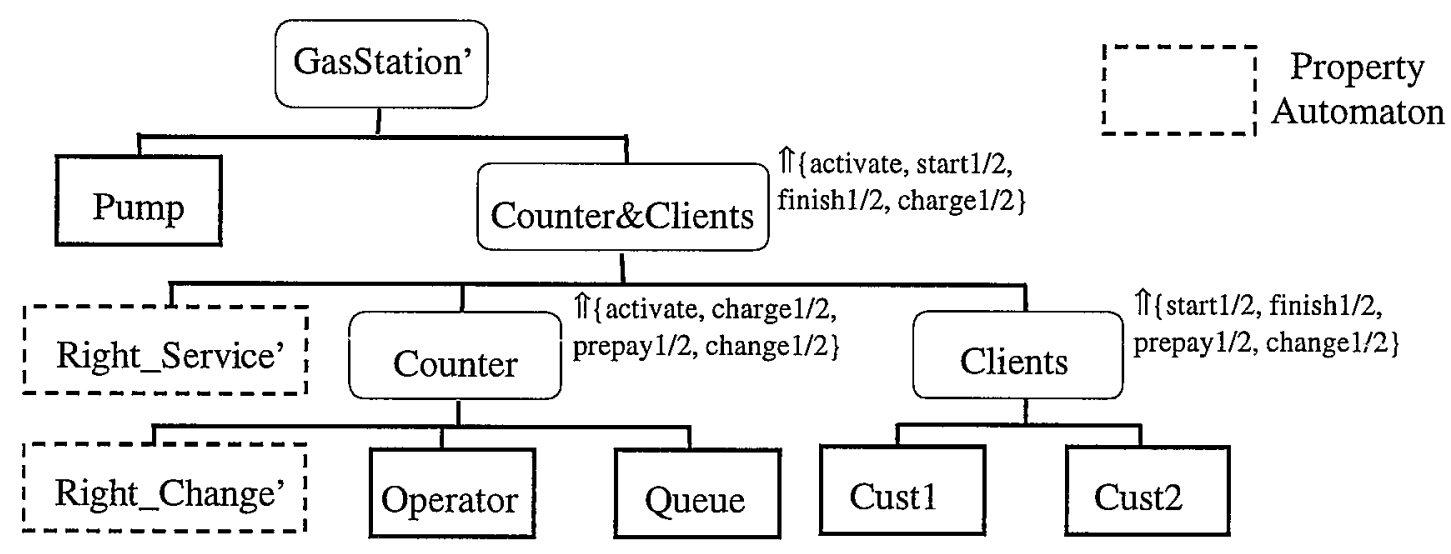

Figure 20: A Re-structured Subsystem Hierarchy of the Gas Control Station for Regression Checking

Suppose Pump is the process selected to be modified to eradicate the reported error. Using the re-structuring algorithm, one can derive a subsystem hierarchy as shown in

Figure 20 for regression checking from that given in Figure 14. The composite LTS Counter\&Clients is constructed in the first round of regression checking and is minimised by hiding all actions not involved in the interaction with Pump. After minimisation, it consists of 48 states and 103 transitions. Subsequent rounds of regression checking can be conducted in one step by composing the LTS of Counter\&Clients and that of the modified Pump. If the LTS derived from the composition of Counter\&Clients and the modified Pump is nontrappable, one can conclude the satisfaction of both properties Right_Change and Right_Service. This is the case if the specification of Pump in Figure 10 is modified to that shown in Figure 21. In fact, the GasStation' formed by composing the Counter\&Clients and the Pump was found to behave like the Pump itself. Figure 22 gives the global LTS based on the subsystem hierarchy in Figure 14 after modification. 


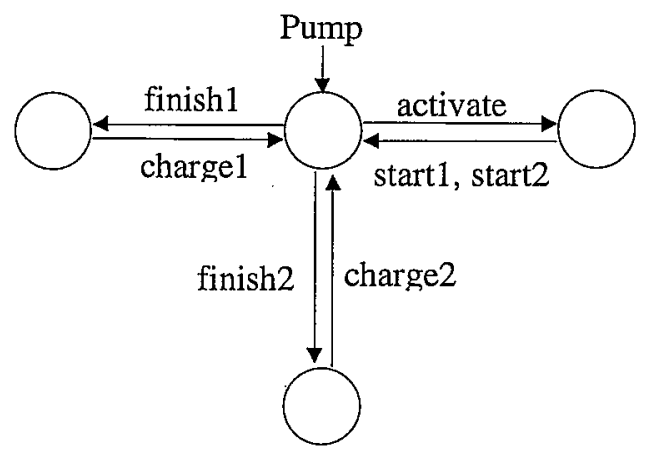

Figure 21: The Modified Pump

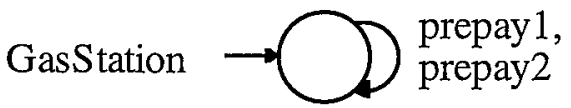

\section{Figure 22: The Global LTS of GasStation}

\subsection{Correctness of the Global LTS}

In previous work [Cheung93], it was shown that the overall behaviour of a system $Z$ remains unchanged after the parallel composition with a process $I f c$ if $Z$ and $I f c$ satisfy the three criteria in an interface theorem (Figure 23). Let $Z \uparrow L$ be a target system and $I f c$ be an image automaton specified by users. Using the construction mechanism for image automata ensures that $I f c$ and $Z$ satisfy criteria (i) and (iii) in the theorem. In addition, it also ensures that $Z \| I f c$ does not contain trapping traces if and only if $\operatorname{tr}(Z \uparrow \alpha I f c) \subseteq \operatorname{tr}(I f c)$. As a result, the absence of reachable trap state $\pi$ in the global LTS of $Z \| I f c$ implies $\operatorname{tr}(Z \uparrow \alpha I f c) \subseteq \operatorname{tr}($ Ifc ), the satisfaction of criteria (ii) in the theorem. Thus a global LTS derived with the inclusion of $I f C$ represents the overall behaviour of $Z$ if the LTS is free from trapping traces ${ }^{4}$. For instance, Figure 22 gives the global LTS constructed with the inclusion of Right_Change' and Right_Service'. Since the LTS does not contain any trapping traces, it represents the overall behaviour of GasStation that satisfies both properties.

4 The use of strong equivalence in the interface theorem allows its utilization for all equivalences that are supersets of the strong equivalence. As weak equivalence is a superset of strong equivalence (c.f. Milner [Milner89a]), the interface theorem is valid for weak equivalence adopted in the modified CRA. 
Suppose $Z$ and $I f c$ are two processes; and $\sim$ denotes the strong equivalence relation.

$$
Z \sim(Z \| I f c)
$$

if

(i) $\alpha I f c \subseteq \alpha Z$;

(ii) $\operatorname{tr}(Z \uparrow \alpha I f c) \subseteq \operatorname{tr}(I f c)$;

(iii) Ifc is a deterministic process free of internal action $\tau$.

\section{Figure 23: Interface Theorem}

\subsection{Early Detection of Infeasible Fixes}

There is an additional benefit in performing re-structuring. Designers may confirm at an early stage if the set of selected processes can be modified to fix the reported errors. For instance, designers may wish to know if modification of the selected processes alone could not help fix the errors. This can be confirmed if the composite LTS formed by the other processes in the system and the property automata after re-structuring contains a trapping trace involving only $\tau$ actions. For example, violation of the safety property cannot be fixed by modifying the behaviour of Pump alone if the composite process Counter\&Clients contains a $\tau$ transition leading to $\pi$ from the initial state. No matter how the designers modify the selected process Pump, the trapping trace remains in the global LTS.

\section{Performance}

A prototype tool based on the concepts of violation and localisation of safety properties has been implemented on the Solaris platform [Giannakopoulou98]. We have applied it to check both the faulty and correct version of the Gas Station system against the properties Right_Service and Right_Change. The performance results of the tool when applied to the faulty and correct versions are given in Figure 24 and Figure 25, respectively. The experiments were conducted on a Sparc Ultra/2 server. Three experiments were performed for each number of customers. The first experiment constructs a global LTS which involves only actions start t $_{i}$ and finish $_{i}$ for checking the property Right_Service. The construction was based on the original subsystem hierarchy in Figure 8. Actions are restricted to each subsystem if they do not participate further in the construction of the global LTS. The result is shown in

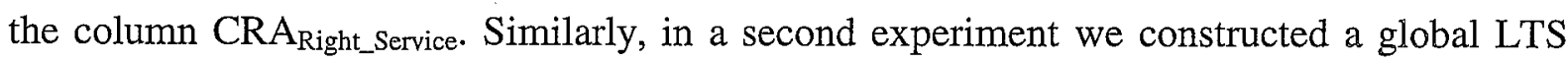
which involved only actions charge $e_{i}$ and changer. Its result is shown in the column 
$\mathrm{CRA}_{\text {Right_Change. }}$ In a third experiment, we applied the proposed enhanced CRA technique and constructed a global LTS consisting of the $\pi$ state and actions prepay. The computational time (in seconds) required in each experiment is given in the associated column $t_{i}$. The column $\Sigma$ s sefore gives the summation of the number of states in all intermediate subsystems constructed in each experiment. The summation is based on the number of states of each subsystem before minimisation.

\begin{tabular}{|c|c|c|c|c|c|c|c|}
\hline \multirow[b]{2}{*}{ Cust\# } & \multicolumn{2}{|c|}{ CRAARight_Service } & \multicolumn{2}{|c|}{ CRA $_{\text {Right_Change }}$} & \multirow[b]{2}{*}{$t_{1}+t_{2}$} & \multicolumn{2}{|c|}{ Enchanced CRA } \\
\hline & $t_{1}(\mathrm{sec})$ & $\sum s_{\text {before }}$ & $\mathrm{t}_{2}(\mathrm{sec})$ & $\Sigma$ Sbefore & & $t_{3}(\sec )$ & $\Sigma s_{\text {before }}$ \\
\hline 2 & 0.3 & 58 & 0.3 & 58 & 0.6 & 0.4 & 70 \\
\hline 3 & 0.5 & 144 & 0.5 & 144 & 1.0 & 0.5 & 190 \\
\hline 4 & 0.6 & 414 & 0.7 & 414 & 1.3 & 0.8 & 552 \\
\hline 5 & 1.5 & 1,352 & 1.5 & 1,352 & 3.0 & 1.8 & 1,740 \\
\hline 6 & 5.3 & 4,802 & 5.4 & 4,802 & 10.7 & 6.2 & 5,862 \\
\hline 7 & 33.6 & 17,932 & 34.4 & 17,932 & 68.0 & 36.0 & 20,758 \\
\hline 8 & 46.8 & 68,950 & 48.1 & 68,950 & 94.9 & 52.1 & 76,300 \\
\hline
\end{tabular}

Figure 24: Computational Effort Taken to Check Properties of the Faulty Gas System

\begin{tabular}{|c|c|c|c|c|c|c|c|}
\hline \multirow[b]{2}{*}{ Cust\# } & \multicolumn{2}{|c|}{$\mathrm{CRA}_{\text {Right_Service }}$} & \multicolumn{2}{|c|}{$\mathrm{CRA}_{\text {Right_Change }}$} & \multirow[b]{2}{*}{$\mathrm{t}_{1}+\mathrm{t}_{2}$} & \multicolumn{2}{|c|}{ Enchanced CRA } \\
\hline & $\mathrm{t}_{1}(\mathrm{sec})$ & $\Sigma S_{\text {before }}$ & $\mathrm{t}_{2}(\mathrm{sec})$ & $\Sigma s_{\text {before }}$ & & $\mathrm{t}_{3}(\mathrm{sec})$ & $\Sigma s_{\text {before }}$ \\
\hline 2 & 0.4 & 74 & 0.3 & 74 & 0.7 & 0.4 & 71 \\
\hline 3 & 0.5 & 180 & 0.5 & 180 & 1.0 & 0.5 & 180 \\
\hline 4 & 0.8 & 480 & 0.8 & 480 & 1.6 & 0.9 & 485 \\
\hline 5 & 1.8 & 1,462 & 1.8 & 1,462 & 3.6 & 2.1 & 1,474 \\
\hline 6 & 5.9 & 4,978 & 5.9 & 4,978 & 11.8 & 6.6 & 4,999 \\
\hline 7 & 35.3 & 18,212 & 35.6 & 18,212 & 70.9 & 37.9 & 18,244 \\
\hline 8 & 48.3 & 69,404 & 50.0 & 69,404 & 98.3 & 52.9 & 69,449 \\
\hline
\end{tabular}

Figure 25: Computational Effort Taken to Check Properties of the Correct Gas System

In these experiments, the computational effort required by the enhanced CRA to check both properties is comparable to that taken for checking a single property. The enhanced CRA technique has an additional advantage that the global LTS involving only actions prepay $_{i}$ can be readily obtained if no property violation is identified. The experiments were made possible with the addition of two optimisations in the mechanism of CRA. Firstly, a subsystem is not minimised if it hides no action. Secondly, the global LTS is not minimised because this is not required for checking safety properties. Since a major fraction of the value in $\Sigma$ sbefore column is contributed by the subsystem Clients which hides no action, the state minimisation 
algorithm need only be applied to a small portion of the states constructed. Otherwise, the experiments for seven and eight customers would consume much more computational time. A possible way to further reduce the computational costs is to combine the enhanced CRA techinique with various state space reduction mechanisms, such as those proposed by Godefroid [Godefroid90] and the previous work of the authors [Cheung93].

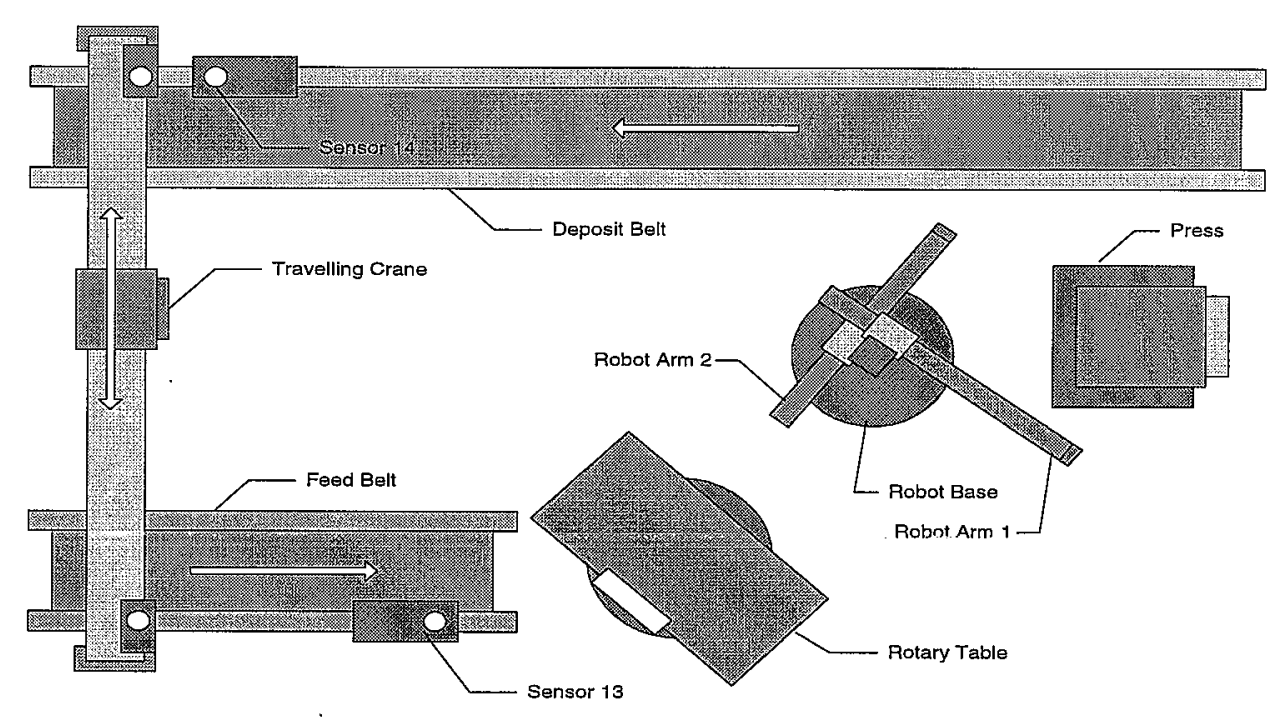

Figure 26: Top View of a Production Cell

To further study the potential of the enhanced CRA, we have applied it to check twenty-one safety properties in a non-trivial production cell system [Lewerentz95]. The production cell system was used as one of the major case studies of the KorSo project [Broy93] to demonstrate the applicability of formal methods for industrial scale systems. A production cell, as shown in Figure 26, forms a basic unit of a real metal-processing plant in Karlsruhe, Germany. Each cell consists of two conveyor belts, a rotary table, a two-armed robot, a press and a travelling crane. When a new blank enters a production cell, it is brought to a rotary table by a feed belt. A two-armed robot moves the blank from the table to a press through robot arm 1 and later moves the forged blank from the press to a deposit belt through robot arm 2 . The deposit belt then conveys the forged blank to a position to be moved out of the cell by a travelling crane.

A list of safety properties was identified in the production cell system [Lewerentz95]. These properties were considered to be the most critical and important requirements amongst all others of the controlling software. In our experiment, the behaviour of a production cell was modelled using an eight layered subsystem hierarchy made up of 19 composite and 29 primitive processes. The safety properties are modelled using 23 property automata. The 
computational effort taken to check the satisfaction of these properties by the production cell system is shown in Figure 27. Some properties are modelled using more than one property automaton, such as Properties 2, 8 and 9. The nature of each property is briefly annotated in the first column of the table. Experiments were conducted on a Sparc Ultra/1 workstation. Full details of the whole case study are available in [Cheung98].

The column $\mathrm{t}_{1}$ shows the computational time taken to construct a global LTS revealing only those actions participating in the corresponding property. The column $\Sigma s_{\text {before }}$ shows the summation of the number of states in the subsystems constructed by CRA before their minimisation. The column $t_{2}$ gives the computational time taken to check a given property of a subsystem after incorporating as its component the associated property. The computation stops when all subsystems to be constructed are made up of only nontrappable components, in which case the prescribed property has been satisfied. All properties, except property 17, are local to some composite processes in the subsystem hierarchy. The results show that most of the twenty-one computations can stop at a stage before the global LTS was constructed. This lead to a roughly $95 \%$ reduction of the total computational effort. Two observations are worth noting from this case study. Firstly, a significant number of safety properties are local to some composite processes. Secondly, local safety properties may be verified without the generation of a global LTS. This can lead to a significant reduction in computational effort.

\begin{tabular}{|c|c|c|c|c|}
\hline \multirow{2}{*}{ Property } & \multicolumn{2}{|c|}{ CRA } & \multicolumn{2}{c|}{ Enhanced CRA } \\
\cline { 2 - 5 } & $\mathrm{t}_{1}(\mathrm{sec})$ & $\Sigma \mathrm{s}_{\text {before }}$ & $\mathrm{t}_{2}(\mathrm{sec})$ & $\Sigma \mathrm{s}_{\text {before }}$ \\
\hline \hline 1 (Robot Rotation) & 140.47 & 1,977 & 5.76 & 822 \\
\hline 2a (Arm Extension) & 62.43 & 1,832 & 5.82 & 807 \\
\hline 2b (Arm Extension) & 88.85 & 1,779 & 5.87 & 807 \\
\hline 3 (Motion of Press) & 33.36 & 1,917 & 6.03 & 815 \\
\hline 4 (Motion of Table) & 29.17 & 1,511 & 5.96 & 806 \\
\hline 5 (Motion of Table) & 30.69 & 1,511 & 5.97 & 811 \\
\hline 6 (Horizontal Motion of Crane) & 40.32 & 1,517 & 6.00 & 806 \\
\hline 7 (Vertical Motion of Crane) & 27.24 & 1,383 & 6.03 & 806 \\
\hline 8a (Press \& Arm1 Collision) & 193.48 & 2,224 & 8.44 & 869 \\
\hline 8b (Press \& Arm1 Collision) & 365.37 & 2,390 & 7.45 & 923 \\
\hline 9a (Press \& Arm2 Collision) & 791.37 & 3,229 & 6.79 & 828 \\
\hline 9b (Press \& Arm2 Collision) & 901.39 & 3,198 & 7.22 & 937 \\
\hline 10 (Lateral Collision of Crane and Deposit Belt) & 15.27 & 1,126 & 7.68 & 950 \\
\hline 11 (Vertical Collision of Crane and 2 Belts) & 15.04 & 1,128 & 7.35 & 929 \\
\hline 12 (Arm1 Deactivation) & 86.64 & 1,859 & 8.23 & 893 \\
\hline 13 (Arm2 Deactivation) & 175.04 & 1,997 & 6.97 & 879 \\
\hline
\end{tabular}




\begin{tabular}{|c|c|c|c|c||}
\hline 14 (Crane Deactivation) & 16.95 & 1,160 & 6.10 & 809 \\
\hline 15 (Transfer of Blanks) & 9.06 & 976 & 4.66 & 732 \\
\hline 16 (Pickup of Blanks) & 29.79 & 1,429 & 5.11 & 779 \\
\hline 17 (Loading of Feed Belt) & 7.34 & 900 & 7.38 & 900 \\
\hline 18 (Loading of Deposit Belt) & 7.22 & 886 & 7.20 & 859 \\
\hline 19 (Loading of Rotary Table) & 14.61 & 1,226 & 6.26 & 818 \\
\hline 20 (Loading of Press) & 15.40 & 1,178 & 6.63 & 829 \\
\hline \hline Total CPU Time Consumed (sec) & $3,096.51$ & & 150.91 & \\
\hline
\end{tabular}

Figure 27: Computational Effort for Checking Properties of a Production Cell System

\section{Conclusion and Future Work}

This paper presents a mechanism to check safety properties associated with subsystems in the framework of CRA techniques. These safety properties are specified in terms of deterministic finite-state machines, called property automata, which may involve actions that are not globally observable. The property automata are said to be violated if the associated subsystems can perform traces not acceptable to them. An image automaton can be derived from each given property automaton. The image automaton transits to and remains in a special trap state $\pi$ when the associated subsystem performs a trace which is not acceptable to the original property automaton. This can be identified directly from the existence of a reachable state $\pi$ in the global LTS. If the LTS is free from state $\pi$, it represents the overall behaviour of the system with no property violations; otherwise the mechanism indicates which safety properties are violated and how they can occur.

To further explore the potential of the technique, we are hoping to apply it to more complex examples. Further work is needed to provide guidance as to which actions to hide and at which point in the subsystem hierarchy to compose the properties to be checked. This is both a logical decision as to which is the most sensible, and an efficiency decision as to which aids the minimisation automation. From the efficiency point of view, the effectiveness of compositional analysis depends on the structure of a subsystem hierarchy. The use of context constraints in [Cheung93, Cheung95] can help to alleviate this problem. These constraints capture behavioural restriction imposed on subsystems by their neighbouring processes. This permits the design of a subsystem hierarchy to be based more on logical than efficiency criteria. Supporting analysis tools [Giannakopoulou98] have been implemented, and we are proposing to incorporate these into an environment for the design and construction of distributed programs, the System Architect's Assistant [Ng96] which utilises the Darwin language [Magee94] for the specification of the software architectures. Finally we are investigating an approach for the specification and checking of liveness properties 
[Cheung97] which would complement the enhanced CRA approach for safety properties described in this paper.

\section{Acknowledgment}

We would like to express our thanks to Dimitra Giannakopoulou for interesting discussions, invaluable suggestions and for her contribution to the analysis tools. We are indebted to Kwok Hung Cheung for his assistance in carrying out a case study of the production cell system. We also wish to express our thanks for financial support for the work by the following grants: HKUST 662/95E, JRS 96/38 and EPSRC Grant GR/J 87022 (TRACTA Project). We would also like to thank the anonymous referees for their careful reading and valuable suggestions.

\section{References}

[Alpern85]B. Alpern and F.B. Schneider. Defining Liveness. Information Processing Letters 21, 4 (October 1985), 181-185.

[Anderson96] R.J. Anderson, P. Beame, S. Burns, W. Chan, F. Modugno, D. Notkin and J.D. Reese. Model Checking Large Software Specification. In Proc. Fourth ACM SIGSOFT Symposium on Foundations of Software Engineering, San Francisco, California, October 1996, pp. 156-166.

[Avrunin91] G.S. Avrunin, U.A. Buy, J.C. Corbett, L.K. Dillon and J.C. Wileden. Automated Analysis of Concurrent Systems with the Constrained Expression Toolset. IEEE Transactions on Software Engineering 17, 11 (1991), 1204-1222.

[Bharadwaj97] R. Bharadwaj and C. Heitmeyer, "Model Checking Complete Requirements Specifications Using Abstraction," Information Technology Division, Naval Research Laboratory NRL/MR/5540-97-7999, November 1997.

[Broy93] M. Broy and S. Jahnichen, "Korrekte Software durch formale Methoden," Technische Universitat Berlin March 1993.

[Cheung98] K.H. Cheung. Compositional Analysis of Complex Distributed Systems, MPhil dissertation, Department of Computer Science, Hong Kong University of Science and Technology, Hong Kong, February 1998.

[Cheung97] S.C. Cheung, D. Giannakopoulou and J. Kramer. Verification of Liveness Properties using Compositional Reachability Analysis. In Proc. ESEC/FSE 97, Zurich, Switzerland, October 1996, pp. 227-243.

[Cheung93] S.C. Cheung and J. Kramer. Enhancing Compositional Reachability Analysis with Context Constraints. In Proc. 1st ACM SIGSOFT International Symposium on the 
Foundations of Software Engineering, ACM SIGSOFT, Los Angeles, California, December 1993, pp. 115-125.

[Cheung94] S.C. Cheung and J. Kramer. Tractable Dataflow Analysis for Distributed Systems. IEEE Transactions on Software Engineering (August 1994), 579-593.

[Cheung95] S.C. Cheung and J. Kramer. Compositional Reachability Analysis of FiniteState Distributed Systems with User-Specified Constraints. In Proc. 3rd ACM SIGSOFT Symposium on the Foundations of Software Engineering, Washington, D.C., October 1995, pp. 140-150.

[Cheung96a] S.C. Cheung and J. Kramer. Checking Subsystem Safety Properties in Compositional Reachability Analysis. In Proc. 18th International Conference on Software Engineering, IEEE, Berlin, Germany, March 1996, pp. 144-154.

[Cheung96b] S.C. Cheung and J. Kramer. Context Constraints for Compositional Reachability Analysis. ACM Transactions on Software Engineering and Methodology (October 1996), 334-377.

[Clarke89] E.M. Clarke, D.E. Long and K.L. McMillan. Compositional Model Checking. In Proc. 4th Annual Symposium on Logic in Computer Science, IEEE, Pacific Grove, California, June 1989, pp. 353-362.

[Corbett95] J.C. Corbett and G.S. Avrunin. Using Integer Programming to Verify General Safety and Liveness Properties. Formal Methods in System Design 6 (January 1995), 97123.

[Corbett96] J.C. Corbett. Evaluating Deadlock Detection Methods for Concurrent Software. IEEE Transactions on Software Engineering 22, 3 (March 1996), 161-180.

[Dwyer97] M.B. Dwyer, V. Carr and L. Hines. Model Checking Graphical User Interfaces using Abstractions. In Proc. ESEC/FSE 97, Zurich, Switzerland, October 1996, pp. 244261.

[Dwyer94]M.B. Dwyer and L.A. Clarke. Data Flow Analysis for Verifying Properties of Concurrent Programs. In Proc. Second ACM SIGSOFT Symposium on Foundations of Software Engineering, New Orleans, Louisiana, USA, December 1994, pp. 62-75.

[Ghezzi91] C. Ghezzi, M. Jazayeri and D. Mandrioli. Fundamentals of Software Engineering, Chapter 6, Prentice-Hall, Inc., (1991).

[Giannakopoulou98] D. Giannakopoulou, J. Kramer and S.C. Cheung. Analysing the Behaviour of Distributed Systems using Tracta. Automated Software Engineering special issue on Automated Analysis of Software (to appear).

[Godefroid90] P. Godefroid. Using Partial Orders to Improve Automatic Verification Methods. In Proc. 2nd International Conference on Computer Aided Verification (CAV), New Brunswick, NJ, USA, June 1990, published in LNCS 531, pp. 176-185. 
[Godefroid91] P. Godefroid and P. Wolper. Using Partial Orders for the Efficient Verification of Deadlock Freedom and Safety Properties. In Proc. 3rd International Conference on Computer Aided Verification (CAV), Aalborg, Denmark, July 1991, published in LNCS 575, pp. 332-342.

[Graf90] S. Graf and B. Steffen. Compositional Minimization of Finite State Systems. In Proc. 2nd International Conference of Computer-Aided Verification, New Brunswick, NJ, USA, June 1990, published in LNCS 531, pp. 186-196.

[Helmbold85] D. Helmbold and D. Luckham. Debugging Ada Tasking Programs. IEEE Software 2, 2 (March 1985), 47-57.

[Hoare85] C.A.R. Hoare. Communicating Sequential Processes, Prentice-Hall, (1985).

[Holzmann91] G. Holzmann. Design and Validation of Computer Protocols, Prentice Hall, Vol. Chapter 8 and 11, (1991).

[Holzmann92] G. Holzmann, P. Godefroid and D. Pirottin. Coverage Preserving Reduction Strategies for Reachability Analysis. In Proc. International Symposium on Protocol Specification, Testing, and Verification (PSTV92), Lake Buena Vista, Florida, NorthHolland, June 1992, pp. 349-364.

[Holzmann97] G.J. Holzmann. The Model Checker SPIN. IFEE Transactions on Software Engineering 23, 5 (May 1997), 279-295.

[Juval98] G. Juval. Specification and Verification of an Object Request Broker. In Proc. International Conference on Software Engineering (ICSE98), Kyoto, Japan, April 1998, pp. 43-52.

[Kemppainen92] J. Kemppainen, M. Levanto, A. Valmari and M. Clegg. "ARA" Puts Advanced Reachability Analysis Techniques Together. In Proc. 5th Nordic Workshop on Programming Environment Research, Programming Environment Research, Tampere. University of Technology, Tampere, Finland, January 1992, pp. 233-257.

[Lewerentz95]C. Lewerentz and T. Lindner. Formal Development of Reactive Systems Case Study Production Cell. In LNCS. Springer-Verlag, 891, 1995.

[Long89] D. Long and L. Clarke. Task Interaction Graphs for Concurrency Analysis. In Proc. 11th International Conference on Software Engineering, Pennsylvania, May 1989, pp. 44-52.

[Magee95] J. Magee, N. Dulay, S. Eisenbach and J. Kramer. Specifying Distributed Software Architectures. In Proc. 5th European Software Engineering Conference (ESEC'95), Sitges, September 1995, published in LNCS 989, pp. 137-153.

[Magee94]J. Magee, N. Dulay and J. Kramer. Regis: A Constructive Development Environment for Distributed Programs. Distributed Systems Engineering Journal 1, 5 (1994), 304-312. 
[Malhotra88] J. Malhotra, S.A. Smolka, A. Giacalone and R. Shapiro. A Tool for Hierarchical Design and Simulation of Concurrent Systems. In Proc. BCS-FACS Workshop on Specification and Verification of Concurrent Systems, Stirling, Scotland, July 1988, pp. 140-152.

[Manna95]Z. Manna and A. Pnueli. Temporal Verification of Reactive Systems: Safety, Springer-Verlag, (1995).

[Mascarenhas98] R. Mascarenhas, D. Karumuri, U. Buy and R. Kenyon. Modeling and Analysis of a Virtual Reality System with Time Petri Nets. In Proc. International Conference on Software Engineering (ICSE98), Kyoto, Japan, April 1998, pp. 33-42.

[Masticola91] S.P. Masticola and B.G. Ryder. A Model of Ada Programs for Static Deadlock Detection in Polynomial Time. In Proc. ACM/ONR Workshop on Parallel and Distributed Debugging, Santa Cruz, California, May 20-21, 1991.

[McMillan93] K.L. McMillan. Symbolic Model Checking, Kluwer Academic Publishers, (1993).

[Milner89a] R. Milner. Communication and Concurrency, Prentice-Hall, (1989).

[Milner89b] R. Milner, J. Parrow and D. Walker, "A Calculus of Mobile Processes Part I and II," University of Edinburgh June 1989.

[Naumovich97] G. Naumovich, G.S. Avrunin, L.A. Clarke and L.J. Osterweil. Applying Static Analysis to Software Architectures. In Proc. ESEC/FSE 97, Zurich, Switzerland, October 1996, pp. 77-93.

[Ng96] K. Ng, J. Kramer, J. Magee and N. Dulay. A Visual Approach to Distributed Programming, Kluwer Academic Publishers, Tools and Environments for Parallel and Distributed Systems, , (1996) pp. 7-31, .

[Olender90] K.M. Olender and L.J. Osterweil. Cecil: A Sequencing Constraint Language for Automatic Static Analysis Generation. IEEE Transactions on Software Engineering 16, 3 (March 1990), 268-280.

[Paige87] R. Paige and R.E. Tarjan. Three Partition Refinement Algorithms. SIAM Journal of Computing 16, 6 (1987), 973-989.

[Peterson81] J.L. Peterson. Petri Net Theory and the Modelling of Systems, Prentice-Hall, (1981).

[Pezzè95] M. Pezzè, R.N. Taylor and M. Young. Graph Models for Reachability Analysis of Concurrent Programs. ACM Transactions on Software Engineering and Methodology 4, 2 (April 1995), 171-213. 
[Rabinovich92] A. Rabinovich. Checking Equivalences Between Concurrent Systems of Finite Agents. In Proc. 19th International Colloquium on Automata, Languages and Programming, Wien, Austria, July 1992, published in LNCS 623, pp. 696-707.

[Reif90] J. Reif and S. Smolka. Data Flow Analysis of Distributed Communicating Processes. International Journal of Parallel Programming 19, 1 (1990), 1-30.

[Sabnani89] K.K. Sabnani, A.M. Lapone and M.Ü. Uyar. An Algorithmic Procedure for Checking Safety Properties of Protocols. IEEE Transactions on Communications 37, 9 (September 1989), 940-948.

[Tai93a] K.C. Tai and P.V. Koppol. Hierarchy-Based Incremental Reachability Analysis of Communication Protocols. In Proc. IEEE International Conference on Network Protocols, San Francisco, California, October 1993, pp. 318-325.

[Tai93b] K.C. Tai and P.V. Koppol. An Incremental Approach to Reachability Analysis of Distributed Programs. In Proc. 7th International Workshop on Software Specification and Design, IEEE, Los Angeles, California, December 1993, pp. 141-150.

[Taylor83] R.N. Taylor. A General-Purpose Algorithm for Analyzing Concurrent Programs. CACM 26 (1983), 362-376.

[Valmari91] A. Valmari. Stubborn Sets for Reduced State Space Generation. In Advances in Petri Nets 90. Springer-Verlag, G. Rozenberg, pp. 491-165, LNCS 483, 1991.

[Valmari92] A. Valmari, "Alleviating State Explosion during Verification of Behavioural Equivalence," Department of Computer Science, University of Helsinki, Finland August 1992.

[Valmari93] A. Valmari, J. Kemppainen, M. Clegg and M. Levanto. Putting advanced reachability analysis techniques together: the 'ARA' tool. In Proc. FME'93: IndustrialStrength Formal Methods. First International Symposium of Formal Methods Europe Proceedings, Odense, Denmark, April 1993, pp. 597-616.

[Yeh91] W.J. Yeh and M. Young. Compositional Reachability Analysis Using Process Algebra. In Proc. Symposium on Testing, Analysis, and Verification (TAV4), ACM SIGSOFT, Victoria, British Columbia, October 1991, pp. 49-59.

[Yeh93a] W.J. Yeh, "Controlling State Explosion in Reachability Analysis," SERC, Purdue University, SERC-TR-147-P, 1993.

[Yeh93b] W.J. Yeh and M. Young, "Hierarchical Tracing of Concurrent Programs," SERC, Purdue University SERC-TR-137-P, 1993.

[Young95] M. Young, R.N. Taylor, D.L. Levine, K.A. Nies and D. Brodbeck. A Concurrency Analysis Tool Suite for Ada Programs: rationale, design, and preliminary experience. ACM Transactions on Software Engineering and Methodology 4, 1 (January 1995), 65106. 